\title{
DESIGN AND SYNTHESIS OF SOME NOVEL 1,2,4-TRIAZOLE-3-YL- MERCAPTO DERIVATIVES AS POTENTIAL ANTI-CANDIDA AGENTS
}

\author{
ANDREEA-IULIA PRICOPIE ${ }^{1}$, LAURIAN VLASE $^{2}$, ADRIAN PIRNĂ ${ }^{3}{ }^{3}$, DAN CRISTIAN \\ VODNAR $^{4}$, GABRIEL MARC $^{1}$, CRISTINA NASTASA ${ }^{1}$, ANCA-MARIA BORCEA ${ }^{1 *}$, IOANA \\ IONUŢ $^{1}$, BRÎNDUȘA TIPERCIUC ${ }^{1}$, OVIDIU ONIGA ${ }^{1}$ \\ ${ }^{I}$ Department of Pharmaceutical Chemistry, "Iuliu Haţieganu" University of Medicine and Pharmacy, 41 Victor Babeș Street, \\ 400012, Cluj-Napoca, Romania \\ ${ }^{2}$ Department of Pharmaceutical Technology and Biopharmaceutics, "Iuliu Haţieganu" University of Medicine and Pharmacy, \\ 41 Victor Babeș Street, 400012, Cluj-Napoca, Romania \\ ${ }^{3}$ National Institute for Research and Development of Isotopic and Molecular Technologies, 67-103 Donath Street, 400293, \\ Cluj-Napoca, Romania \\ ${ }^{4}$ Department of Food Science and Technology, University of Agricultural Sciences and Veterinary Medicine, 3-5 Mănăștur \\ Street, 400372, Cluj-Napoca, Romania
}

*corresponding author: borcea.anca@umfcluj.ro

Manuscript received: May 2018

\begin{abstract}
In the context of the alarming incidence of the multidrug-resistant Candida $s p$. based infections, a new series of 1,2,4triazole-3-yl-mercapto derivatives were synthesized and evaluated as potential antifungal agents. The affinity of the synthesized compounds towards the catalytic site of the lanosterol 14 $\alpha$-demethylase (CYP51) was evaluated in silico, by molecular docking studies. The antifungal activity of the titled compounds was evaluated in vitro against pathogenic strains of Candida sp., by measuring the minimum inhibitory concentration (MIC) and the minimum fungicidal concentration (MFC). The data obtained from the docking simulation showed that the new synthesized compounds might act as noncovalent inhibitors of fungal CYP51. The results of the in vitro antifungal screening support their potential anti-Candida activity, compound $\mathbf{3 b}$ exhibiting a similar effect as fluconazole, used as antifungal reference drug.
\end{abstract}

\section{Rezumat}

În contextul creșterii alarmante a numărului de infecții cauzate de specii de Candida multirezistente, au fost sintetizați și evaluați ca potențiali agenți antifungici noi compuși cu structură 3-mercapto-1,2,4-triazolică. A fost evaluată in silico, prin studii de andocare moleculară, afinitatea de legare a noilor derivaţi triazolici de situsul catalitic al lanosterol $14 \alpha$-demetilazei fungice (CYP51). Activitatea antifungică a compușilor sintetizați a fost evaluată in vitro, pe tulpini standardizate de Candida $s p$., prin determinarea concentrației minime inhibitorii (CMI) și a concentrației minime fungicide (CMF). Rezultatele testării in silico au confirmat potențialul anti-Candida al compușilor noi sintetizați, aceștia acționând ca inhibitori ai lanosterol $14 \alpha-$ demetilazei fungice, legarea de enzimă fiind mediată de interacțiuni non-covalente. Potențialul antifungic al noilor derivați triazolici este susținut și de rezultatele testării in vitro, compusul 3b având CMI egală cu cea a fluconazolului, folosit ca compus de referință.

Keywords: antifungal, azole derivatives, molecular docking, lanosterol $14 \alpha$-demethylase

\section{Introduction}

Candida sp. based infections, ranging from superficial and mucosal to systemic and bloodstream ones, are the most frequently reported fungal infections in Europe and USA [3], being associated with significant morbidity and mortality, as a result of the increasing resistance of the pathogenic fungal strains to the currently available antifungals [28]. Azoles have been widely used as potent anti-Candida drugs [21]. Their molecular mechanism of action is based on the inhibition of lanosterol $14 \alpha$-demethylase (CYP51), a key enzyme involved in the fungal ergosterol biosynthesis [7]. Due to the high affinity of the nucleophilic nitrogen of the azole heterocycle towards the heme $\mathrm{Fe}^{2+}$, these compounds are responsible for the cross-over inhibition of human CYP51 and other CYP450 enzymes [22]. Safety issues related to the azole drugs, such as hepatotoxicity, endocrine disorders, drug interactions, unfavourable pharmacokinetic properties and fungistatic activity, corroborated with the increasing resistance to treatment of the fungal strains, require the development of new CYP51 inhibitors, with greater selectivity towards this fungal enzyme [6, 26, 27].

A particular interest in the field of antifungal drug design was assigned to the 1,2,4-triazole heterocyclic compounds [9]. Their aromaticity and electron-rich property [25] enable them to bind lanosterol $14 \alpha-$ 
FARMACIA, 2018, Vol. 66, 6

demethylase, both through covalent coordination of the heme $\mathrm{Fe}^{2+}$ by the $\mathrm{N} 4$ nitrogen atom of the triazole ring and non-covalent interactions, including $\pi-\pi$ stacking [23]. 3-S-substituted-1,2,4-triazole ring systems have also been studied during the last years and the antifungal activity has been reported for a large number of derivatives $[10,12]$. Moreover, Slinker was reported to improve important drug-like parameters, such as lipophilicity and water solubility [13].

Considering all the above, we synthesized a new series of 1,2,4-triazole-3-yl-mercapto derivatives starting from thymol, a terpenoid phenol, based on the evidences related to its anti-Candida properties $[1,2$, 17, 20]. A molecular docking study was carried out in order to evaluate the interaction of the synthesized compounds with the catalytic site of fungal lanosterol $14 \alpha$-demethylase, as well as their ability to trigger the cross-over inhibition of the human target enzyme. The antifungal activity of the synthesized compounds was evaluated in vitro, against pathogenic strains of Candida sp.

\section{Materials and Methods}

\section{Chemistry}

All chemicals and reagents used for synthesis were of analytical grade purity and were obtained from Alfa Aesar (Karlsruhe, Germany) and Merck (Darmstadt, Germany). Analytical thin layer chromatography (TLC) carried out on Silica Gel $60 \mathrm{~F}_{254}$ sheets was used for monitoring the reaction progress and to confirm the purity of the newly synthesized compounds. A mixture of ethyl acetate:n-hexane 3:1 was used as elution system, UV light $(254 \mathrm{~nm})$ being employed for visualization. The uncorrected melting points were determined with an Electrothermal melting point meter through the open glass capillary method. In order to confirm the structures of the synthesized compounds, spectral analytical methods (mass spectrometry [MS], infrared spectroscopy [IR], nuclear magnetic resonance [NMR]) and elemental analysis were used. IR spectra were recorded on a Jasco FT/IR 6100 spectrometer (Jasco, Easton, MD), using anhydrous potassium bromide for sample preparation. MS analyses were performed in positive ionization at $70 \mathrm{eV}$, using an Agilent 1100 series and an Agilent Ion Trapp SL mass spectrometer (Agilent, Santa Clara, CA). ${ }^{1} \mathrm{H}-$ NMR spectra were recorded on a Bruker Advance NMR spectrometer (Karlsruhe, Germany), operating at $500 \mathrm{MHz}$, using DMSO- $d_{6}$ as solvent and tetramethylsilane (TMS) as internal standard. ${ }^{13} \mathrm{C}-\mathrm{NMR}$ analyses were performed on a Bruker Advance NMR spectrometer, operating at $125 \mathrm{MHz}$, in DMSO- $d_{6}$, using a Waltz-16 decoupling scheme, with TMS as internal standard. Chemical shift $(\delta)$ values were expressed in parts per million (ppm). For the elemental analyses, a Vario El CHNS instrument was used.
General procedure for the synthesis of 2-(2-(2-isopropyl-5-methylphenoxy)acetyl)-N-substitutedhydrazinecarbothioamide (2a-c)

Equimolar quantities of acetohydrazide (1) $(10 \mathrm{mmol}$, $2.22 \mathrm{~g})$ and $N$-substituted isothiocyanate $(10 \mathrm{mmol})$ in absolute ethanol $(30 \mathrm{~mL})$ were refluxed for 6 hours. After cooling to the room temperature, the reaction mixture was poured into ice cold water. The obtained precipitate was filtered and crystallized from water:ethanol 3:1 mixture.

General procedure for the synthesis of 5-(2-isopropyl-5-methylphenoxy)-4-substituted-4H-1,2,4triazole-3-thiol (3a-c)

The alcoholic solution of the 2 a-c $(1 \mathrm{mmol}$ in $3 \mathrm{~mL}$ of absolute ethanol) was treated with triethylamine (15 drops) and the reaction mixture was refluxed for 5 hours. The solvent was removed under vacuum and the obtained precipitate was filtered, dried and recrystallized from ethanol.

General procedure for the synthesis of 1-((5-(2-isopropyl-5-methylphenoxy)-4-substituted-4H-1,2,4triazol-3-yl)thio)ethers (4a-o)

A mixture of 3a-c $(1 \mathrm{mmol})$ and potassium hydroxide (1.5 mmol, $0.084 \mathrm{~g}$ ) in $5 \mathrm{~mL} N, N$-dimethylformamide:methanol 1:1 was stirred at room temperature for 1 hour. Then, an equimolar quantity $(1 \mathrm{mmol})$ of $\alpha$-halocarbonyl compound was added and the stirring was continued for other 4 hours. After the reaction was complete (according to the TLC detection) the mixture was poured over ice cold water. The formed precipitate was filtered, dried and recrystallized from ethanol, to afford the target compounds.

Molecular Docking Study

In order to evaluate the affinity of the synthesized compounds towards Candida albicans lanosterol $14 \alpha-$ demethylase, as well as to assess their ability for cross-over inhibition of the human homologous, a molecular docking study was carried out using AutoDock 4.2.6. [15]. Fluconazole was used as reference drug. The sequence homologous (PBD ID: 5EQB) used as template for the building of the fungal lanosterol $14 \alpha$-demethylase was chosen from the Protein Data Bank based on the results of a BLAST search. The fungal target enzyme (5EQBCA), was built through homology modelling based on the UniProt P10613 sequence from Candida albicans (www.uniprot.org), using SWISS-MODEL (www.swissmodel.expasy.org). The crystal structure of human CYP51 was taken from the Protein Data Bank (PDB ID: 3JUS) [4]. Dataset files containing the ligands and target enzymes (5EQBCA and 3JUS) were prepared using a previously reported protocol $[5,14]$.

AutoDock uses a Lamarckian genetic algorithm [15], based on the random modifications of structural parameters of the ligand, in order to identify the most likely binding conformation, corresponding to a solution of minimum energy. The binding energy of possible ligands poses is evaluated through a grid- 
based method. The conformational search space is configured as a cubic area, with edges $x=y=z=76$ points and centre Cartesian coordinates set to $\mathrm{x}=$ $39.048, y=4.888, z=51.406$ for the human target enzyme and to $\mathrm{x}=23.134, \mathrm{y}=13.943, \mathrm{z}=19.959$ for the fungal homologous. The space between the grid points was set to $0.375 \AA$. Visualization and analysis of the docking results were performed using UCSF Chimera 1.10.2 [16] and AutoDockTools 1.5.6. [15]. The values of the inhibition constants $\left(\mathrm{K}_{\mathrm{i}}\right)$ were calculated through the conversion of the binding energy values $(\Delta \mathrm{G})$, using formula $K_{\mathrm{i}}=\mathrm{e}^{\frac{\Delta \mathrm{G} \times 1000}{\mathrm{R} \times \mathrm{T}}}$, where $\mathrm{R}$ represents the Regnault constant $=1.98719 \frac{\mathrm{kcal}}{\mathrm{Kx} \mathrm{mol}}$ and $\mathrm{T}=298.15 \mathrm{~K}$.

Antifungal activity assay

The antifungal activity of the synthesized compounds was evaluated in vitro against three pathogenic strains of Candida sp. The microorganisms used for this assay were Candida albicans ATCC 10231, Candida albicans ATCC 18804 and Candida krusei ATCC 6258 and were obtained from the Food Biotechnology Laboratory, Life Sciences Institute, University of Agricultural Sciences and Veterinary Medicine ClujNapoca, Romania. The minimum inhibitory concentration (MIC) and the minimum fungicidal concentration (MFC) were determined according to the Clinical and Laboratory Standards Institute guidelines, following a previously reported protocol $[14,18]$. Fluconazole was used as reference antifungal drug. All experiments were performed in triplicate.

\section{Results and Discussion}

\section{Chemistry}

The synthesis of the titled compounds was carried out performing a stepwise reaction protocol, as outlined in Figure 1. The key intermediate hydrazide (1) was obtained using a previously reported synthesis protocol [19]. The 1,2,4-triazole-3-yl-mercapto intermediates 3a-c were synthesized, according to the literature [11], through the condensation of the hydrazide compound 1 with various $N$-substituted isothiocyanates, followed by intramolecular cyclization, in basic media, of the corresponding $\mathrm{N}$-substituted acyl-thiosemicarbazide derivatives 2a-c. The target 1,2,4-triazole-3yl-mercapto derivatives were obtained through a mild $S$-alkylation reaction, by stirring, at room temperature, the compounds 3a-c with different $\alpha$-halocarbonyl compounds, using a mixture of methanol: $N, N$,-dimethylformamide $1: 1$ as solvent. The progress of the reaction was monitored by TLC. The synthesized compounds were purified by recrystallization from the appropriate solvents and their physico-chemical and spectral properties were investigated.

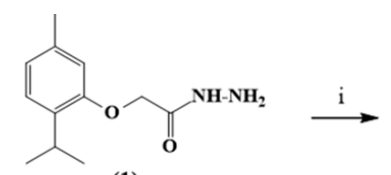

(1)

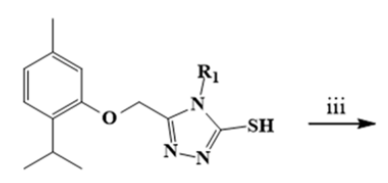

(3a-c)

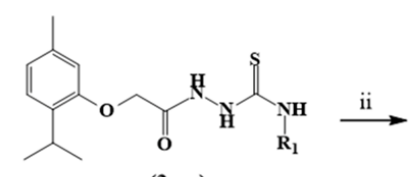

(2a-c)

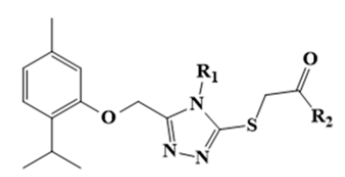

(4a-0)

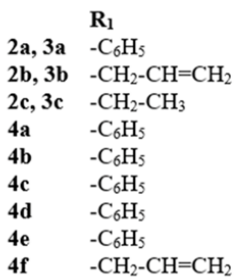

$\mathbf{R}_{\mathbf{2}}$

$-$

$-$

$-\mathrm{NH}_{2}$

$-\mathrm{CH}_{3}$

$-\mathrm{C}_{6} \mathrm{H}_{5}$

$-\mathrm{C}_{6} \mathrm{H}_{4}-4-\mathrm{Cl}$

$-\mathrm{C}_{6} \mathrm{H}_{3}-3-\mathrm{C}$

$-\mathrm{CH}_{2}-\mathrm{CH}=\mathrm{CH}_{2}-\mathrm{NH}_{2}$
$\mathbf{R}_{1}$

$-\mathrm{CH}_{2}-\mathrm{CH}=\mathrm{CH}_{2}-\mathrm{CH}_{3}$

$-\mathrm{CH}_{2}-\mathrm{CH}=\mathrm{CH}_{2}-\mathrm{C}_{6} \mathrm{H}$

$-\mathrm{CH}_{2}-\mathrm{CH}=\mathrm{CH}_{2}-\mathrm{C}_{6} \mathrm{H}_{4}-4-\mathrm{Cl}$

$-\mathrm{CH}_{2}-\mathrm{CH}=\mathrm{CH}_{2}-\mathrm{C}_{6} \mathrm{H}_{3}-3-\mathrm{CONH}_{2}-4-\mathrm{OH}$

$\begin{array}{lll}-\mathrm{CH}_{2}-\mathrm{CH}_{3} & -\mathrm{NH}_{2}\end{array}$

$\begin{array}{ll}-\mathrm{CH}_{2}-\mathrm{CH}_{3} & -\mathrm{NH}_{2} \\ -\mathrm{CH}_{2}-\mathrm{CH}_{3} & -\mathrm{CH}_{3}\end{array}$

$4 \mathrm{~m}-\mathrm{CH}_{2}-\mathrm{CH}_{3} \quad-\mathrm{C}_{6} \mathrm{H}_{3}$

$4 n-\mathrm{CH}_{2}-\mathrm{CH}_{3} \quad-\mathrm{C}_{6} \mathrm{H}_{4}-4-\mathrm{Cl}$

$40-\mathrm{CH}_{2}-\mathrm{CH}_{3}$

$-\mathrm{C}_{6} \mathrm{H}_{3}-3-\mathrm{CONH}_{2}-4-\mathrm{OH}$

Figure 1.

Synthesis of 1,2,4-triazole-3-yl-mercapto derivatives

i. $\mathrm{R}_{1}-\mathrm{N}=\mathrm{C}=\mathrm{S} / \mathrm{EtOH}$ abs./reflux; ii.TEA/EtOH abs./ reflux; iii. $\mathrm{X}-\mathrm{CH}_{2}-\mathrm{CO}-\mathrm{R}_{2} / \mathrm{KOH} / \mathrm{MeOH}$ :DMF 1:1

The structural assignments of the synthesized compounds were based on their spectral data (MS, IR, ${ }^{1} \mathrm{H}-\mathrm{NMR}$ and $\left.{ }^{13} \mathrm{C}-\mathrm{NMR}\right)$ and elemental analysis.

2-(2-(2-isopropyl-5-methylphenoxy)acetyl)-N-phenylhydrazinecarbothioamide (2a):

Analytical calculated for $\mathrm{C}_{19} \mathrm{H}_{23} \mathrm{~N}_{3} \mathrm{O}_{2} \mathrm{~S}(\%)$ : C, 63.84; H, 6.49; N, 11.75; S, 8.97; Found (\%): C, 63.93; H,
6.38; N, 11.93; S, 8.71; white powder; m.p. 172 $173^{\circ} \mathrm{C}$; yield $91 \%$; IR(KBr) $v_{\max } \mathrm{cm}^{-1}: 3295(\mathrm{~N}-\mathrm{H})$, 3095 (C-H arom.), $1674(\mathrm{C}=\mathrm{O}), 1272(\mathrm{C}=\mathrm{S}), 1244$ (C-O-C); MS (EI, $70 \mathrm{eV}): \mathrm{m} / \mathrm{z}=358.2[\mathrm{M}+\mathrm{H}]^{+} ;{ }^{1} \mathrm{H}-$ NMR (500 MHz, DMSO- $\left.d_{6}, \delta / \mathrm{ppm}\right): 7.90$ (s, 2H, -NH-CS-NH-), 7.82 (s, 1H, -NH-NH), 7.43 (t, 1H ArH), 7.41 (t, 2H, ArH), 7.39 - 7.38 (d, 2H, ArH), 
FARMACIA, 2018, Vol. 66, 6

7.03 (d, 1H, ArH), 6.79 (s, 1H, ArH), 6.75 (d, 1H, ArH), 4.98 (s, 2H, Ar-O-CH $\left.\mathrm{CH}_{2}\right), 2.95$ (m, 1H, Ar-CH- $\left.\left(\mathrm{CH}_{3}\right)_{2}\right)$, $2.24\left(\mathrm{~s}, 3 \mathrm{H}, \mathrm{Ar}-\mathrm{CH}_{3}\right), 1.01\left(\mathrm{~d}, 6 \mathrm{H}, \mathrm{Ar}-\mathrm{CH}-\left(\mathrm{CH}_{3}\right)_{2}\right)$; ${ }^{13} \mathrm{C}-\mathrm{NMR}$ (125 MHz, DMSO-d $\left.d_{6}, \delta / \mathrm{ppm}\right): 182.75$ $(\mathrm{C}=\mathrm{S}), 165.34(\mathrm{C}=\mathrm{O}), 155.52,136.41,134.48,133.96$, $130.05,129.81,128.27,126.05,122.71,113.51$ (aromatic ring C), $60.96\left(-\mathrm{O}-\mathrm{CH}_{2}\right), 25.98\left(\mathrm{Ar}-\mathrm{CH}-\left(\mathrm{CH}_{3}\right)_{2}\right), 23.18$ (Ar-CH- $\left.\left(\mathbf{C H}_{3}\right)_{2}\right), 20.99\left(\mathrm{Ar}-\mathbf{C H}_{3}\right)$.

$\mathrm{N}$-allyl-2-(2-(2-isopropyl-5-methylphenoxy)acetyl)hydrazinecarbothioamide (2b):

Analytical calculated for $\mathrm{C}_{16} \mathrm{H}_{23} \mathrm{~N}_{3} \mathrm{O}_{2} \mathrm{~S}$ (\%): C, 59.78; H, 7.21; N, 13.07; S, 9.98; Found (\%): C, 59.93; H, 7.14; N, 13.21; S, 9.81; white powder; m.p. 142 $143^{\circ} \mathrm{C}$; yield 75\%; IR(KBr) $v_{\max } \mathrm{cm}^{-1}: 3268(\mathrm{~N}-\mathrm{H})$, 3081 (C-H arom.), $3054(=\mathrm{C}-\mathrm{H}), 1659(\mathrm{C}=\mathrm{O}), 1266$ $(\mathrm{C}=\mathrm{S}), 1243$ (C-O-C); MS (EI, $70 \mathrm{eV}): \mathrm{m} / \mathrm{z}=322.2$ $[\mathrm{M}+\mathrm{H}]^{+} ;{ }^{1} \mathrm{H}-\mathrm{NMR}\left(500 \mathrm{MHz}, \mathrm{DMSO}-d_{6}, \delta / \mathrm{ppm}\right)$ : 7.14 (s, 2H, -NH-CS-NH-), 7.12 (s, 1H, -NH-NH), $7.10(\mathrm{~d}, 1 \mathrm{H}, \mathrm{ArH}), 6.79$ (d, 1H, ArH), 5.97 (m, 1H, $\left.-\mathrm{CH}=\mathrm{CH}_{2}\right), 5.23\left(\mathrm{~s}, 2 \mathrm{H}, \mathrm{Ar}-\mathrm{O}-\mathrm{CH}_{2}\right), 5.01-4.97$ and $4.94\left(\mathrm{~d}, 1 \mathrm{H}\right.$ each, $\left.=\mathrm{CH}_{2}\right), 4.92\left(\mathrm{~d}, 2 \mathrm{H},-\mathrm{CH}_{2}-\right), 3.15(\mathrm{~m}$, $\left.1 \mathrm{H}, \mathrm{Ar}-\mathrm{CH}-\left(\mathrm{CH}_{3}\right)_{2}\right), 2.28$ (s, 3H, Ar- $\left.\mathrm{CH}_{3}\right), 1.11-1.09$ (d, 6H, Ar-CH- $\left.\left(\mathrm{CH}_{3}\right)_{2}\right) ;{ }^{13} \mathrm{C}-\mathrm{NMR}\left(125 \mathrm{MHz}\right.$, DMSO- $d_{6}$, $\delta / p p m): 180.09(\mathrm{C}=\mathrm{S}), 166.34(\mathrm{C}=\mathrm{O}), 154.15,136.34$, 133.65 (aromatic ring $\mathrm{C}), 131.26(-\mathrm{CH}=), 126.18,122.52$ (aromatic ring $\mathrm{C}$ ), $118.23\left(=\mathrm{CH}_{2}\right), 113.44$ (aromatic ring $\mathrm{C}), 60.38\left(-\mathrm{O}-\mathrm{CH}_{2}\right), 46.37\left(-\mathrm{CH}_{2}-\right), 25.92(\mathrm{Ar}-$ $\left.\mathbf{C H}-\left(\mathrm{CH}_{3}\right)_{2}\right), 23.39\left(\mathrm{Ar}-\mathrm{CH}-\left(\mathbf{C H}_{3}\right)_{2}\right), 21.29\left(\mathrm{Ar}-\mathbf{C H}_{3}\right)$. $\mathrm{N}$-ethyl-2-(2-(2-isopropyl-5-methylphenoxy)acetyl)hydrazinecarbothioamide (2c):

Analytical calculated for $\mathrm{C}_{15} \mathrm{H}_{23} \mathrm{~N}_{3} \mathrm{O}_{2} \mathrm{~S}$ (\%): C, 58.22; H, 7.49; N, 13.58; S, 10.36; Found (\%): C, 58.49; H, 7.37 ; N, 13.42; S, 10.55; white powder; m.p. 117 $118^{\circ} \mathrm{C}$; yield $78 \%$; IR(KBr) $v_{\max } \mathrm{cm}^{-1}: 3316(\mathrm{~N}-\mathrm{H})$, 3089 (C-H arom.), $1681(\mathrm{C}=\mathrm{O}), 1274(\mathrm{C}=\mathrm{S}), 1253$ (C-O-C); MS (EI, $70 \mathrm{eV}): \mathrm{m} / \mathrm{z}=310.3[\mathrm{M}+\mathrm{H}]^{+} ;{ }^{1} \mathrm{H}-$ NMR (500 MHz, DMSO- $\left.d_{6}, \delta / \mathrm{ppm}\right): 7.63(\mathrm{~s}, 1 \mathrm{H}$, -NH-NH), 7.55 (s, 2H, -NH-CS-NH-), 7.11 (d, 1H, ArH), 6.91 (s,1H, ArH), 6.83 (d, 1H, ArH), 5.20 (s, $\left.2 \mathrm{H}, \mathrm{Ar}-\mathrm{O}-\mathrm{CH}_{2}\right), 4.06\left(\mathrm{~m}, 2 \mathrm{H},-\mathrm{CH}_{2}-\right), 3.22(\mathrm{~m}, 1 \mathrm{H}$, $\left.\mathrm{Ar}-\mathrm{CH}-\left(\mathrm{CH}_{3}\right)_{2}\right), 2.30$ (s, 3H, Ar- $\left.\mathrm{CH}_{3}\right), 1.25$ (t, 3H, $\left.-\mathrm{CH}_{3}\right), 1.12$ (d, 6H, Ar-CH- $\left.\left(\mathrm{CH}_{3}\right)_{2}\right) ;{ }^{13} \mathrm{C}-\mathrm{NMR}(125$ MHz, DMSO- $\left.d_{6}, \delta / \mathrm{ppm}\right): 181.54(\mathrm{C}=\mathrm{S}), 167.45(\mathrm{C}=\mathrm{O})$, $154.95,136.53,133.79,126.34,122.60,113.46$ (aromatic ring $\mathrm{C}), 60.71\left(-\mathrm{O}-\mathrm{CH}_{2}\right), 39.06\left(-\mathrm{CH}_{2}-\right), 26.07(\mathrm{Ar}-$ CH- $\left.\left(\mathrm{CH}_{3}\right)_{2}\right), 23.40\left(\mathrm{Ar}-\mathrm{CH}-\left(\mathrm{CH}_{3}\right)_{2}\right), 21.46\left(\mathrm{Ar}-\mathrm{CH}_{3}\right)$, $13.89\left(-\mathrm{CH}_{3}\right)$.

5-(2-isopropyl-5-methylphenoxy)-4-phenyl-4H-1,2,4triazole-3-thiol (3a):

Analytical calculated for $\mathrm{C}_{19} \mathrm{H}_{21} \mathrm{~N}_{3} \mathrm{OS}(\%)$ : $\mathrm{C}, 67.23$; H, 6.24; N, 12.38; S, 9.45; Found (\%): C, 66.91; H, 6.12 ; N, 12.65; S, 9.61; white powder; m.p. 174 $175^{\circ} \mathrm{C}$; yield $69 \%$; IR( $\left.\mathrm{KBr}\right) v_{\max } \mathrm{cm}^{-1}: 3044(\mathrm{C}-\mathrm{H}$ arom), 2546 (S-H), 1616 (-N=CH-), 1255 (C-O-C); MS (EI, $70 \mathrm{eV}): \mathrm{m} / \mathrm{z}=340.2[\mathrm{M}+\mathrm{H}]^{+} ;{ }^{1} \mathrm{H}-\mathrm{NMR}$ (500 MHz, DMSO- $\left.d_{6}, \delta / \mathrm{ppm}\right): 14.05$ (s, 1H, -SH), 7.55 (t, 1H, ArH), 7.54 - 7.50 (t, 2H, ArH), 7.46 - 7.45 (d, 2H, ArH), $7.01-6.99(\mathrm{~d}, 1 \mathrm{H}, \mathrm{ArH}), 6.72(\mathrm{~s}, 1 \mathrm{H}$,
$\mathrm{ArH}), 6.70$ (d, 1H, ArH), $4.98\left(\mathrm{~s}, 2 \mathrm{H}, \mathrm{Ar}-\mathrm{O}-\mathrm{CH}_{2}\right)$, $2.78\left(\mathrm{~m}, 1 \mathrm{H}, \mathrm{Ar}-\mathrm{CH}-\left(\mathrm{CH}_{3}\right)_{2}\right), 2.20\left(\mathrm{~s}, 3 \mathrm{H}, \mathrm{Ar}-\mathrm{CH}_{3}\right)$, 0.97 - 0.96 (d, 6H, Ar-CH- $\left.\left(\mathrm{CH}_{3}\right)_{2}\right) ;{ }^{13} \mathrm{C}-\mathrm{NMR}(125$ $\left.\mathrm{MHz}, \mathrm{DMSO}-d_{6}, \delta / \mathrm{ppm}\right): 167.81\left(\mathrm{C}_{3}\right.$-triazole ring), 155.38 (aromatic ring $\mathrm{C}), 154.46\left(\mathrm{C}_{2}\right.$-triazole ring), 136.32, 134.04, 129.91, 129.73, 128.25, 126.19, 122.63, 113.43 (aromatic ring $\mathrm{C}), 60.81\left(-\mathrm{O}-\mathrm{CH}_{2}\right), 25.64$ (Ar$\left.\mathbf{C H}-\left(\mathrm{CH}_{3}\right)_{2}\right), 23.27$ (Ar-CH- $\left.\left(\mathrm{CH}_{3}\right)_{2}\right), 21.29\left(\mathrm{Ar}-\mathbf{C H}_{3}\right)$. 4-allyl-5-(2-isopropyl-5-methylphenoxy)-4H-1,2,4triazole-3-thiol (3b):

Analytical calculated for $\mathrm{C}_{16} \mathrm{H}_{21} \mathrm{~N}_{3} \mathrm{OS}(\%)$ : C, 63.33; H, 6.9; N, 13.85; S, 10.57; Found (\%): C, 63.71; H, 7.10; N, 13.51; S, 10.69; white powder; m.p. 134 $135^{\circ} \mathrm{C}$; yield $72 \%$; IR( $\left.\mathrm{KBr}\right) v_{\max } \mathrm{cm}^{-1}: 3092(\mathrm{C}-\mathrm{H}$ arom), 3053 (=C-H), $2574(\mathrm{~S}-\mathrm{H}), 1616(-\mathrm{N}=\mathrm{CH}-)$, 1248 (C-O-C); MS (EI, $70 \mathrm{eV}): \mathrm{m} / \mathrm{z}=304.1[\mathrm{M}+\mathrm{H}]^{+}$; ${ }^{1} \mathrm{H}-\mathrm{NMR}\left(500 \mathrm{MHz}, \mathrm{DMSO}-d_{6}, \delta / \mathrm{ppm}\right): 13.93$ (s, 1H, -SH), $7.09-7.08$ (d, 1H, ArH), 6.98 (s, 1H, ArH), $6.78-6.76(\mathrm{~d}, 1 \mathrm{H}, \mathrm{ArH}), 5.93\left(\mathrm{~m}, 1 \mathrm{H}, \mathrm{CH}=\mathrm{CH}_{2}\right), 5.21$ (s, 2H, Ar-O-CH $\left.\mathrm{CH}_{2}\right), 4.99-4.95$ and $4.90(\mathrm{~d}, 1 \mathrm{H}$ each, $\left.=\mathrm{CH}_{2}\right), 4.72\left(\mathrm{~d}, 2 \mathrm{H},-\mathrm{CH}_{2}-\right), 3.14\left(\mathrm{~m}, 1 \mathrm{H}, \mathrm{Ar}-\mathrm{CH}-\left(\mathrm{CH}_{3}\right)_{2}\right)$, 2.27 (s, 3H, Ar- $\left.\mathrm{CH}_{3}\right), 1.09-1.08$ (d, 6H, Ar-CH- $\left.\left(\mathrm{CH}_{3}\right)_{2}\right)$; ${ }^{13} \mathrm{C}-\mathrm{NMR}$ (125 MHz, DMSO- $\left.d_{6}, \delta / \mathrm{ppm}\right): 164.07\left(\mathrm{C}_{3^{-}}\right.$ triazole ring), 154.92 (aromatic ring $\mathrm{C}$ ), $152.31\left(\mathrm{C}_{2^{-}}\right.$ triazole ring), 136.35, 132.21 (aromatic ring $\mathrm{C}$ ), 131.25 $(-\mathrm{CH}=), 126.22,122.47$ (aromatic ring C), $118.28\left(=\mathrm{CH}_{2}\right)$, 113.40 (aromatic ring $\mathrm{C}), 60.34\left(-\mathrm{O}-\mathrm{CH}_{2}\right), 46.44$ $\left(-\mathrm{CH}_{2}-\right), 25.95\left(\mathrm{Ar}-\mathrm{CH}-\left(\mathrm{CH}_{3}\right)_{2}\right), 23.30\left(\mathrm{Ar}-\mathrm{CH}-\left(\mathrm{CH}_{3}\right)_{2}\right)$, $21.38\left(\mathrm{Ar}-\mathrm{CH}_{3}\right)$.

4-ethyl-5-(2-isopropyl-5-methylphenoxy)-4H-1,2,4triazole-3-thiol (3c):

Analytical calculated for $\mathrm{C}_{15} \mathrm{H}_{21} \mathrm{~N}_{3} \mathrm{OS}(\%)$ : C, 61.82; H, 7.26; N, 14.42; S 11.00; Found (\%): C, 62.08; H, 7.14; N, 14.67; S, 10.74; white powder; m.p. 139 $140^{\circ} \mathrm{C}$; yield $75 \%$; IR( $\left.\mathrm{KBr}\right) v_{\max } \mathrm{cm}^{-1}: 3044(\mathrm{C}-\mathrm{H}$ arom), 2553 (S-H), 1613 (-N=CH-), 1253 (C-O-C); MS (EI, $70 \mathrm{eV}): \mathrm{m} / \mathrm{z}=292.1[\mathrm{M}+\mathrm{H}]^{+} ;{ }^{1} \mathrm{H}-\mathrm{NMR}(500 \mathrm{MHz}$, DMSO- $\left.d_{6}, \delta / \mathrm{ppm}\right): 13.84$ (s, 1H, -SH) 7.12 - $7.10(\mathrm{~d}$, $1 \mathrm{H}, \mathrm{ArH}), 6.97$ (s, 1H, ArH), 6.80 - 6.79 (d,1H, ArH), $5.18\left(\mathrm{~s}, 2 \mathrm{H}, \mathrm{Ar}-\mathrm{O}-\mathrm{CH}_{2}\right), 4.08-4.03\left(\mathrm{~m}, 2 \mathrm{H},-\mathrm{CH}_{2}-\right)$, $3.16\left(\mathrm{~m}, 1 \mathrm{H}, \mathrm{Ar}-\mathrm{CH}-\left(\mathrm{CH}_{3}\right)_{2}\right), 2.28\left(\mathrm{~s}, 3 \mathrm{H}, \mathrm{Ar}-\mathrm{CH}_{3}\right)$, 1.27 (t, 3H, - $\left.\mathrm{CH}_{2}-\mathrm{CH}_{3}\right), 1.11-1.10$ (d, 6H, Ar-CH$\left.\left(\mathrm{CH}_{3}\right)_{2}\right) ;{ }^{13} \mathrm{C}-\mathrm{NMR}\left(125 \mathrm{MHz}, \mathrm{DMSO}-d_{6}, \delta / \mathrm{ppm}\right)$ : $167.57\left(\mathrm{C}_{3}\right.$-triazole ring), 154.91 (aromatic ring $\mathrm{C}$ ), 148.57 ( $\mathrm{C}_{2}$-triazole ring), 136.50, 133.85, 126.38, 122.66, 113.54 (aromatic ring $\mathrm{C}), 60.67\left(-\mathrm{O}-\mathrm{CH}_{2}\right), 39.19$ $\left(-\mathrm{CH}_{2}-\right), 26.08\left(\mathrm{Ar}-\mathrm{CH}-\left(\mathrm{CH}_{3}\right)_{2}\right), 23.31\left(\mathrm{Ar}-\mathrm{CH}-\left(\mathrm{CH}_{3}\right)_{2}\right)$, $21.41\left(\mathrm{Ar}-\mathrm{CH}_{3}\right), 13.84\left(-\mathrm{CH}_{3}\right)$.

2-((5-(2-isopropyl-5-methylphenoxy)-4-phenyl-4H1,2,4-triazol-3-yl)thio)acetamide (4a):

Analytical calculated for $\mathrm{C}_{21} \mathrm{H}_{24} \mathrm{~N}_{4} \mathrm{O}_{2} \mathrm{~S}(\%)$ : C, 63.61; H, 6.10; N, 14.13; S 8.09; Found (\%): C, 63.32; H, 6.29 ; N, 13.90; S, 8.31; white powder; m.p. $168-169^{\circ} \mathrm{C}$; yield $84 \%$; IR( $\mathrm{KBr}) v_{\max } \mathrm{cm}^{-1}: 3318(\mathrm{~N}-\mathrm{H}$ amide), 3058 (C-H arom), $1673(\mathrm{C}=\mathrm{O}$ amide), $1610(-\mathrm{N}=\mathrm{CH}-)$, 1252 (C-O-C); MS (EI, $70 \mathrm{eV}): \mathrm{m} / \mathrm{z}=397.2[\mathrm{M}+\mathrm{H}]^{+}$; ${ }^{1} \mathrm{H}-\mathrm{NMR}$ (500 MHz, DMSO-d $\left.d_{6}, \delta / \mathrm{ppm}\right): 7.67$ (s, 2H, -CO-NH2), 7.58 (t, 1H, ArH), 7.57 (t, 2H, ArH), 7.52 - 
FARMACIA, 2018, Vol. 66, 6

7.50 (d,2H, ArH), 7.00 - 6.98 (d, 1H, ArH), 6.78 (s, 1H, $\mathrm{ArH}), 6.71$ - 6.70 (d, 1H, ArH), 5.10 (s, 2H, Ar-O-CH $)_{2}$, $3.95\left(\mathrm{~s}, 2 \mathrm{H},-\mathrm{S}-\mathrm{CH}_{2}\right), 2.80$ (m, $\left.1 \mathrm{H}, \operatorname{Ar}-\mathrm{CH}-\left(\mathrm{CH}_{3}\right)_{2}\right)$, 2.21 (s, 3H, Ar-CH $)$, 0.94 - 0.93 (d, 6H, Ar-CH- $\left.\left(\mathrm{CH}_{3}\right)_{2}\right)$; ${ }^{13} \mathrm{C}-\mathrm{NMR}$ (125 MHz, DMSO- $\left.d_{6}, \delta / \mathrm{ppm}\right): 168.92(\mathrm{C}=\mathrm{O})$, 155.48 (aromatic ring $\mathrm{C}$ ), $154.67\left(\mathrm{C}_{3}\right.$-triazole ring), $152.26\left(\mathrm{C}_{2}\right.$-triazole ring), 136.29, 133.96, 133.24, $130.46,130.28,127.21,126.11,122.44,113.42$ (aromatic ring $\mathrm{C}), 60.53\left(-\mathrm{O}-\mathrm{CH}_{2}\right), 36.36\left(-\mathrm{S}-\mathrm{CH}_{2}-\right), 25.59(\mathrm{Ar}-$ $\left.\mathbf{C H}-\left(\mathrm{CH}_{3}\right)_{2}\right), 23.28\left(\mathrm{Ar}-\mathrm{CH}-\left(\mathrm{CH}_{3}\right)_{2}\right), 21.32\left(\mathrm{Ar}-\mathbf{C H}_{3}\right)$. 1-((5-(2-isopropyl-5-methylphenoxy)-4-phenyl-4H1,2,4-triazol-3-yl)thio)propan-2-one (4b):

Analytical calculated for $\mathrm{C}_{22} \mathrm{H}_{25} \mathrm{~N}_{3} \mathrm{O}_{2} \mathrm{~S}(\%)$ : C, 66.81; H, 6.37; N, 10.62; S, 8.11; Found (\%): C, 67.03; H, 6.61 ; N, 10.38; S, 7.97; white powder; m.p. $121-122^{\circ} \mathrm{C}$; yield $41 \%$; IR( $\mathrm{KBr}) v_{\max } \mathrm{cm}^{-1}: 3052$ (C-H arom), 1713 $(\mathrm{C}=\mathrm{O}), 1612(-\mathrm{N}=\mathrm{CH}-), 1254$ (C-O-C); MS (EI, $70 \mathrm{eV})$ : $\mathrm{m} / \mathrm{z}=396.5[\mathrm{M}+\mathrm{H}]^{+} ;{ }^{1} \mathrm{H}-\mathrm{NMR}\left(500 \mathrm{MHz}\right.$, DMSO- $d_{6}$, $\delta / \mathrm{ppm}): 7.63$ (d, 2H, ArH), 7.44 (d, 2H, ArH), 7.39 (t, 1H, ArH), $7.02(\mathrm{~d}, 1 \mathrm{H}, \mathrm{ArH}), 6.79(\mathrm{~s}, 1 \mathrm{H}, \mathrm{ArH})$, $6.73(\mathrm{~d}, 1 \mathrm{H}, \mathrm{ArH}), 5.07$ (s, 2H, Ar-O-CH $), 4.12(\mathrm{~s}$, $\left.2 \mathrm{H},-\mathrm{S}-\mathrm{CH}_{2}\right), 3.01$ (m, $\left.1 \mathrm{H}, \mathrm{Ar}-\mathrm{CH}-\left(\mathrm{CH}_{3}\right)_{2}\right), 2.31$ (s, $3 \mathrm{H},-\mathrm{CO}-\mathrm{CH}_{3}$ ), 2.23 (s, 3H, Ar- $\left.\mathrm{CH}_{3}\right), 0.95-0.94$ (d, $\left.6 \mathrm{H}, \mathrm{Ar}-\mathrm{CH}-\left(\mathrm{CH}_{3}\right)_{2}\right) ;{ }^{13} \mathrm{C}-\mathrm{NMR}$ (125 MHz, DMSO- $d_{6}$, $\delta / \mathrm{ppm})$ : $197.65(\mathrm{C}=\mathrm{O}), 155.39$ (aromatic ring $\mathrm{C}$ ), 154.81 ( $\mathrm{C}_{3}$-triazole ring), 151.97 ( $\mathrm{C}_{2}$-triazole ring), 136.40, 134.09, 133.89, 133.20, 130.17, 130.05, 126.21, 122.61, 113.49 (aromatic ring $\mathrm{C}), 60.91\left(-\mathrm{O}-\mathrm{CH}_{2}\right), 37.01$ $\left(-\mathrm{S}_{-}-\mathrm{CH}_{2}-\right), 26.21\left(-\mathrm{CH}_{3}\right), 25.49\left(\mathrm{Ar}-\mathrm{CH}-\left(\mathrm{CH}_{3}\right)_{2}\right), 23.72$ (Ar-CH- $\left.\left(\mathrm{CH}_{3}\right)_{2}\right), 20.99\left(\mathrm{Ar}-\mathrm{CH}_{3}\right)$;

2-((5-(2-isopropyl-5-methylphenoxy)-4-phenyl-4H1,2,4-triazol-3-yl)thio)-1-phenylethanone (4c):

Analytical calculated for $\mathrm{C}_{27} \mathrm{H}_{27} \mathrm{~N}_{3} \mathrm{O}_{2} \mathrm{~S}(\%)$ : C, 70.87; H, 5.95; N, 9.18; S, 7.01; Found (\%): C, 70.61; H, 5.83; N, 9.42; S, 7.12; light yellow powder; m.p. $104-105^{\circ} \mathrm{C}$; yield $61 \%$; IR(KBr) $v_{\max } \mathrm{cm}^{-1}: 3059$ (C-H arom), $1681(\mathrm{C}=\mathrm{O}), 1613(-\mathrm{N}=\mathrm{CH}-), 1253$ (C-O-C); MS (EI, $70 \mathrm{eV}): \mathrm{m} / \mathrm{z}=458.4[\mathrm{M}+\mathrm{H}]^{+} ;{ }^{1} \mathrm{H}-$ NMR (500 MHz, DMSO- $\left.d_{6}, \delta / p p m\right): 8.06$ - 8.04 (d, $2 \mathrm{H}, \mathrm{ArH}), 7.67$ (d, 2H, ArH), 7.63 - 7.62 (t, 1H, ArH), $7.61(\mathrm{t}, 1 \mathrm{H}, \operatorname{ArH}), 7.53(\mathrm{~d}, 2 \mathrm{H}, \operatorname{ArH}), 7.51(\mathrm{~d}, 2 \mathrm{H}, \operatorname{ArH})$, $7.06(\mathrm{~d}, 1 \mathrm{H}, \mathrm{ArH}), 6.81(\mathrm{~s}, 1 \mathrm{H}, \mathrm{ArH}), 6.75(\mathrm{~d}, 1 \mathrm{H}$, ArH), 4.99 (s, 2H, Ar-O-CH $), 4.33$ (s, 2H, -S-CH $)$, $2.78\left(\mathrm{~m}, 1 \mathrm{H}, \mathrm{Ar}-\mathrm{CH}-\left(\mathrm{CH}_{3}\right)_{2}\right), 2.22\left(\mathrm{~s}, 3 \mathrm{H}, \mathrm{Ar}-\mathrm{CH}_{3}\right)$, 0.97 - 0.95 (d, 6H, Ar-CH- $\left.\left(\mathrm{CH}_{3}\right)_{2}\right) ;{ }^{13} \mathrm{C}-\mathrm{NMR}(125 \mathrm{MHz}$, DMSO- $\left.d_{6}, \delta / \mathrm{ppm}\right): 191.98(\mathrm{C}=\mathrm{O}), 155.42$ (aromatic ring $\mathrm{C}), 154.61\left(\mathrm{C}_{3}\right.$-triazole ring $), 152.20\left(\mathrm{C}_{2}\right.$-triazole ring), 136.44, 134.25, 133.92, 130.47, 130.12, 129.74, $129.48,129.04,128.17,127.26,126.11,122.50,113.41$ (aromatic ring $\mathrm{C}), 60.93\left(-\mathrm{O}-\mathrm{CH}_{2}\right), 36.95\left(-\mathrm{S}-\mathrm{CH}_{2}-\right)$, $25.54\left(\mathrm{Ar}-\mathrm{CH}-\left(\mathrm{CH}_{3}\right)_{2}\right), 23.30\left(\mathrm{Ar}-\mathrm{CH}-\left(\mathrm{CH}_{3}\right)_{2}\right), 21.14$ $\left(\mathrm{Ar}-\mathrm{CH}_{3}\right)$.

1-(4-chlorophenyl)-2-((5-(2-isopropyl-5-methylphenoxy)4-phenyl-4H-1,2,4-triazol-3-yl)thio)ethanone (4d): Analytical calculated for $\mathrm{C}_{27} \mathrm{H}_{26} \mathrm{ClN}_{3} \mathrm{O}_{2} \mathrm{~S}(\%)$ : C, 65.91; H, 5.33; N, 8.54; S, 6.52; Found (\%): C, 65.46; H, 5.11 ; N, 8.87; S, 6.31; yellow powder; m.p. 108 $109^{\circ} \mathrm{C}$; yield $89 \%$; IR(KBr) $v_{\max } \mathrm{cm}^{-1}: 3053(\mathrm{C}-\mathrm{H}$ arom), $1680(\mathrm{C}=\mathrm{O}), 1614(-\mathrm{N}=\mathrm{CH}-), 1253(\mathrm{C}-\mathrm{O}-\mathrm{C})$, 627 (C-Cl); MS (EI, $70 \mathrm{eV}): \mathrm{m} / \mathrm{z}=492.7[\mathrm{M}+\mathrm{H}]^{+}$; ${ }^{1} \mathrm{H}-\mathrm{NMR}$ (500 MHz, DMSO- $d_{6}, \delta / \mathrm{ppm}$ ): $8.05-8.03$ (d, 2H, ArH), 7.65 - 7.63 (d, 2H, ArH), 7.59 - 7.58 (d, 2H, ArH), 7.52 (d, 2H, ArH), 7.50 (t, 1H, ArH), 7.00 - $6.98(\mathrm{~d}, 1 \mathrm{H}, \mathrm{ArH}), 6.77$ (s, 1H, ArH), 6.71 $6.70(\mathrm{~d}, 1 \mathrm{H}, \mathrm{ArH}), 5.09$ (d, 2H, Ar-O-CH $\left.\mathrm{CH}_{2}\right), 4.37$ (s, $\left.2 \mathrm{H},-\mathrm{S}-\mathrm{CH}_{2}\right), 2.75\left(\mathrm{~m}, 1 \mathrm{H}, \mathrm{Ar}-\mathrm{CH}-\left(\mathrm{CH}_{3}\right)_{2}\right), 2.20$ (s, $\left.3 \mathrm{H}, \mathrm{Ar}-\mathrm{CH}_{3}\right), 0.95-0.93$ (d, 6H, Ar-CH- $\left.\left(\mathrm{CH}_{3}\right)_{2}\right)$; ${ }^{13} \mathrm{C}-\mathrm{NMR}$ (125 MHz, DMSO- $\left.d_{6}, \delta / \mathrm{ppm}\right): 192.25(\mathrm{C}=\mathrm{O})$, 155.47 (aromatic ring $\mathrm{C}), 154.78\left(\mathrm{C}_{3}\right.$-triazole ring), $152.36\left(\mathrm{C}_{2}\right.$-triazole ring), 136.31, 134.49, 133.96, $133.23,130.80,130.52$, 129.71, 129.43, 128.30, 127.21, $126.17,122.54,113.42$ (aromatic ring $\mathrm{C}$ ), 60.54 (-O$\left.\mathrm{CH}_{2}\right), 36.52$ (-S-CH $\left.2^{-}\right), 25.74\left(\mathrm{Ar}-\mathrm{CH}-\left(\mathrm{CH}_{3}\right)_{2}\right), 23.32$ $\left(\mathrm{Ar}-\mathrm{CH}-\left(\mathrm{CH}_{3}\right)_{2}\right), 21.43\left(\mathrm{Ar}-\mathrm{CH}_{3}\right)$.

2-hydroxy-5-(2-((5-(2-isopropyl-5-methylphenoxy)-4phenyl-4H-1,2,4-triazol-3-yl)thio)acetyl)benzamide $(4 e)$ :

Analytical calculated for $\mathrm{C}_{28} \mathrm{H}_{28} \mathrm{~N}_{4} \mathrm{O}_{4} \mathrm{~S}$ (\%): C, 65.10; H, 5.46; N, 10.85; S, 6.21; Found (\%): C, 65.43; H, 5.58; N, 10.53; S, 6.43; light yellow powder; m.p. $119-120^{\circ} \mathrm{C}$; yield $61 \%$; IR(KBr) $v_{\max } \mathrm{cm}^{-1}: 3354$ (N-H amide), 3074 (C-H arom), $1675(\mathrm{C}=\mathrm{O}$ ketone), 1671 (C=O amide), 1632 (-N=CH-), $1383(\mathrm{O}-\mathrm{H}), 1253$ (C-O-C); MS (EI, $70 \mathrm{eV}): \mathrm{m} / \mathrm{z}=517.4[\mathrm{M}+\mathrm{H}]]^{+} ;{ }^{1} \mathrm{H}-$ NMR (500 MHz, DMSO- $\left.d_{6}, \delta / \mathrm{ppm}\right): 12.15$ (s, $\left.1 \mathrm{H},-\mathrm{OH}\right)$, 8.23 - 8.21 (d, 1H, ArH), 8.18 (d, 1H, ArH), 8.15 (s, $\left.2 \mathrm{H},-\mathrm{CO}-\mathrm{NH}_{2}\right), 7.87$ (t, 2H, ArH), 7.60 (t, 1H, ArH), 7.54 (d, 2H, ArH), 7.51 (d, 1H, ArH), 7.07 (d, 1H, ArH), 6.82 (s, 1H, ArH), 6.78 (d, 1H, ArH), 5.00 (s, 2H, Ar-O- $\left.\mathrm{CH}_{2}\right), 4.39$ (s, 2H, -S-CH $), 2.81$ (m, 1H, Ar$\left.\mathrm{CH}-\left(\mathrm{CH}_{3}\right)_{2}\right), 2.26$ (s, 3H, Ar- $\left.\mathrm{CH}_{3}\right), 1.00-0.97$ (d, 6H, Ar-CH- $\left.\left(\mathrm{CH}_{3}\right)_{2}\right) ;{ }^{13} \mathrm{C}-\mathrm{NMR}$ (125 MHz, DMSO- $d_{6}$, $\delta / \mathrm{ppm}): 192.40(\mathrm{C}=\mathrm{O}), 167.62$ (aromatic ring $\mathrm{C}), 167.45$ ( $\mathrm{C}=\mathrm{O}$ amide), 155.52 (aromatic ring $\mathrm{C}), 154.49\left(\mathrm{C}_{3}\right.$ triazole ring), 152.18 ( $\mathrm{C}_{2}$-triazole ring), 136.39, 134.31, $133.99,131.85,130.74,129.68,129.24,128.22$, 127.95 , $126.17,122.54,116.98,115.21,113.42$ (aromatic ring C), 60.87 (-O-CH 2$), 36.78$ (-S-CH $\left.2_{2}\right), 25.61$ (Ar-CH$\left.\left(\mathrm{CH}_{3}\right)_{2}\right), 23.27\left(\mathrm{Ar}-\mathrm{CH}-\left(\mathrm{CH}_{3}\right)_{2}\right), 21.24\left(\mathrm{Ar}-\mathbf{C H}_{3}\right)$. 2-((4-allyl-5-(2-isopropyl-5-methylphenoxy)-4H-1,2,4triazol-3-yl)thio)acetamide (4f):

Analytical calculated for $\mathrm{C}_{18} \mathrm{H}_{24} \mathrm{~N}_{4} \mathrm{O}_{2} \mathrm{~S}(\%)$ : C, 59.87; $\mathrm{H}, 6.71$; N, 15.54; S, 8.90; Found (\%): C, 60.18; H, $6.59 ; \mathrm{N}, 15.33$; S, 9.08; white powder; m.p. $162-163^{\circ} \mathrm{C}$; yield $89 \%$; IR(KBr) $v_{\max } \mathrm{cm}^{-1}: 3301(\mathrm{~N}-\mathrm{H}$ amide), 3154 (C-H arom), 3052 ( $=\mathrm{C}-\mathrm{H}), 1677(\mathrm{C}=\mathrm{O}$ amide), 1612 (-N=CH-), 1254 (C-O-C); MS (EI, $70 \mathrm{eV})$ : $\mathrm{m} / \mathrm{z}=361.2[\mathrm{M}+\mathrm{H}]^{+} ;{ }^{1} \mathrm{H}-\mathrm{NMR}\left(500 \mathrm{MHz}\right.$, DMSO- $d_{6}$, $\delta / \mathrm{ppm}): 7.31\left(\mathrm{~s}, 2 \mathrm{H},-\mathrm{CO}-\mathrm{NH}_{2}\right), 7.09$ (d, 1H, ArH), $6.96(\mathrm{~s}, 1 \mathrm{H}, \mathrm{ArH}), 6.77$ (d, 1H, ArH), $5.90(\mathrm{~m}, 1 \mathrm{H}$, $\left.\mathrm{CH}=\mathrm{CH}_{2}\right), 5.17\left(\mathrm{~s}, 2 \mathrm{H}, \mathrm{Ar}-\mathrm{O}-\mathrm{CH}_{2}\right), 4.94-4.93$ and $4.91\left(\mathrm{~d}, 1 \mathrm{H}\right.$ each, $\left.=\mathrm{CH}_{2}\right), 4.76\left(\mathrm{~d}, 2 \mathrm{H},-\mathrm{CH}_{2}\right), 3.88(\mathrm{~s}$, $\left.2 \mathrm{H},-\mathrm{S}-\mathrm{CH}_{2}\right), 3.12$ (m, 1H, Ar-CH- $\left.\left(\mathrm{CH}_{3}\right)_{2}\right), 2.29$ (s, $\left.3 \mathrm{H}, \mathrm{Ar}-\mathrm{CH}_{3}\right), 1.09-1.08$ (d, 6H, Ar-CH- $\left.\left(\mathrm{CH}_{3}\right)_{2}\right)$; ${ }^{13} \mathrm{C}-\mathrm{NMR}$ (125 MHz, DMSO- $\left.d_{6}, \delta / \mathrm{ppm}\right): 169.05$ $(\mathrm{C}=\mathrm{O}), 154.22$ (aromatic ring $\mathrm{C}), 152.35\left(\mathrm{C}_{2}\right.$-triazole 
FARMACIA, 2018, Vol. 66, 6

ring), 151.22 ( $\mathrm{C}_{3}$-triazole ring), 136.29, 132.77 (aromatic ring $\mathrm{C}$ ), $131.19(-\mathrm{CH}=), 126.23,122.49$ (aromatic ring C), $118.17\left(=\mathrm{CH}_{2}\right), 113.35$ (aromatic ring $\mathrm{C}$ ), 60.46 $\left(-\mathrm{O}-\mathrm{CH}_{2}\right), 46.39\left(-\mathrm{CH}_{2}-\right), 41.05\left(-\mathrm{S}_{-} \mathrm{CH}_{2}-\right), 25.97(\mathrm{Ar}-$ $\left.\mathbf{C H}-\left(\mathrm{CH}_{3}\right)_{2}\right), 23.41\left(\mathrm{Ar}-\mathrm{CH}-\left(\mathbf{C H}_{3}\right)_{2}\right), 21.40\left(\mathrm{Ar}-\mathbf{C H}_{3}\right)$. 1-((4-allyl-5-(2-isopropyl-5-methylphenoxy)-4H-1,2,4triazol-3-yl)thio)propan-2-one (4g):

Analytical calculated for $\mathrm{C}_{19} \mathrm{H}_{25} \mathrm{~N}_{3} \mathrm{O}_{2} \mathrm{~S}(\%)$ : C, 63.48; H, 7.01; N, 11.69; S, 8.92; Found (\%): C, 63.26; H, 7.15; N, 11.46; S, 9,11; light brown powder; m.p. $57-58^{\circ} \mathrm{C}$; yield $61 \%$; \%; IR(KBr) $v_{\max } \mathrm{cm}^{-1}: 3049$ (C-H arom), 3029 (=C-H), $1705(\mathrm{C}=\mathrm{O}), 1613(-\mathrm{N}=\mathrm{CH}-)$, 1256 (C-O-C); MS (EI, $70 \mathrm{eV}): \mathrm{m} / \mathrm{z}=360.2[\mathrm{M}+\mathrm{H}]^{+}$; ${ }^{1} \mathrm{H}-\mathrm{NMR}\left(500 \mathrm{MHz}, \mathrm{DMSO}-d_{6}, \delta / \mathrm{ppm}\right): 7.03$ (d, $1 \mathrm{H}$, ArH), 6.95 (s, 1H, ArH), 6.74 (d,1H, ArH), 5.95 (m, $\left.1 \mathrm{H}, \mathrm{CH}=\mathrm{CH}_{2}\right), 5.16\left(\mathrm{~s}, 2 \mathrm{H}, \mathrm{Ar}-\mathrm{O}-\mathrm{CH}_{2}\right), 4.93-4.90$ $\left(\mathrm{d}, 1 \mathrm{H}\right.$ each, $\left.=\mathrm{CH}_{2}\right), 4.74\left(\mathrm{~d}, 2 \mathrm{H},-\mathrm{CH}_{2}\right), 3.90(\mathrm{~s}, 2 \mathrm{H}$, $\left.-\mathrm{S}-\mathrm{CH}_{2}\right), 3.17\left(\mathrm{~m}, 1 \mathrm{H}, \mathrm{Ar}-\mathrm{CH}-\left(\mathrm{CH}_{3}\right)_{2}\right), 2.35$ (s, 3H, $\mathrm{CO}-\mathrm{CH}_{3}$ ), 2.30 (s, 3H, Ar- $\mathrm{CH}_{3}$ ), 1.08 - 1.07 (d, 6H, $\left.\mathrm{Ar}-\mathrm{CH}-\left(\mathrm{CH}_{3}\right)_{2}\right) ;{ }^{13} \mathrm{C}-\mathrm{NMR}$ (125 MHz, DMSO-d $d_{6}$, $\delta / p p m): 199.05(\mathrm{C}=\mathrm{O}), 154.42$ (aromatic ring $\mathrm{C}), 152.45$ ( $\mathrm{C}_{2}$-triazole ring), $151.19\left(\mathrm{C}_{3}\right.$-triazole ring), 136.21, 132.79 (aromatic ring $\mathrm{C}), 131.10(-\mathrm{CH}=), 126.34,122.51$ (aromatic ring C), $118.13\left(=\mathrm{CH}_{2}\right), 113.09$ (aromatic ring $\mathrm{C}), 60.51\left(-\mathrm{O}-\mathrm{CH}_{2}\right), 46.51\left(-\mathrm{CH}_{2}-\right), 41.12$ (-S$\left.\mathrm{CH}_{2}-\right), 25.88\left(\mathrm{Ar}-\mathrm{CH}-\left(\mathrm{CH}_{3}\right)_{2}\right), 23.11\left(\mathrm{Ar}-\mathrm{CH}-\left(\mathrm{CH}_{3}\right)_{2}\right)$, $21.29\left(\mathrm{Ar}-\mathrm{CH}_{3}\right)$;

2-((4-allyl-5-(2-isopropyl-5-methylphenoxy)-4H-1,2,4triazol-3-yl)thio)-1-phenylethanone (4h):

Analytical calculated for $\mathrm{C}_{24} \mathrm{H}_{27} \mathrm{~N}_{3} \mathrm{O}_{2} \mathrm{~S}(\%)$ : C, 68.38; H, 6.46; N, 9.97; S, 7.61; Found (\%): C, 68.15; H, 6.71; $\mathrm{N}, 10.14$; S, 7.38; white powder; m.p. $134-135^{\circ} \mathrm{C}$; yield $32 \%$; IR(KBr) $v_{\max } \mathrm{cm}^{-1}: 3053$ (C-H arom), 3027 (=C-H), $1687(\mathrm{C}=\mathrm{O}), 1611(-\mathrm{N}=\mathrm{CH}-), 1252(\mathrm{C}-\mathrm{O}-\mathrm{C})$; MS (EI, $70 \mathrm{eV}): \mathrm{m} / \mathrm{z}=422.4[\mathrm{M}+\mathrm{H}]^{+} ;{ }^{1} \mathrm{H}-\mathrm{NMR}(500$ MHz, DMSO- $\left.d_{6}, \delta / \mathrm{ppm}\right): 7.97$ (d, 2H, ArH), 7.94 (d, 2H, ArH), 7.78 (t, 1H, ArH), 7.05 (d, 1H, ArH), 6.95 (s, 1H, ArH), 6.80 (d, 1H, ArH), $5.92(\mathrm{~m}, 1 \mathrm{H}$, $\left.\mathrm{CH}=\mathrm{CH}_{2}\right), 5.11\left(\mathrm{~s}, 2 \mathrm{H}, \mathrm{Ar}-\mathrm{O}-\mathrm{CH}_{2}\right), 4.95-4.93(\mathrm{~d}, 1 \mathrm{H}$ each, $\left.=\mathrm{CH}_{2}\right), 4.80\left(\mathrm{~d}, 2 \mathrm{H},-\mathrm{CH}_{2}-\right), 3.92\left(\mathrm{~s}, 2 \mathrm{H},-\mathrm{S}_{-}-\mathrm{CH}_{2}\right)$, $3.13\left(\mathrm{~m}, 1 \mathrm{H}, \mathrm{Ar}-\mathrm{CH}-\left(\mathrm{CH}_{3}\right)_{2}\right), 2.28\left(\mathrm{~s}, 3 \mathrm{H}, \mathrm{Ar}-\mathrm{CH}_{3}\right)$, $1.09-1.08$ (d, 6H, Ar-CH- $\left.\left(\mathrm{CH}_{3}\right)_{2}\right) ;{ }^{13} \mathrm{C}-\mathrm{NMR}(125$ $\left.\mathrm{MHz}, \mathrm{DMSO}-d_{6}, \delta / \mathrm{ppm}\right): 190.13(\mathrm{C}=\mathrm{O}), 154.42$ (aromatic ring $\mathrm{C}), 152.65\left(\mathrm{C}_{2}\right.$-triazole ring $), 151.58\left(\mathrm{C}_{3}\right.$-triazole ring), 136.21, 132.89 (aromatic ring $\mathrm{C}), 131.27(-\mathrm{CH}=)$, 131.10, 129.38, 129.06, 127.53, 126.34, 122.51 (aromatic ring $\mathrm{C}$ ), $118.29\left(=\mathrm{CH}_{2}\right), 113.09$ (aromatic ring $\mathrm{C}$ ), 60.39 $\left(-\mathrm{O}-\mathrm{CH}_{2}\right), 46.33\left(-\mathrm{CH}_{2}-\right), 41.10\left(-\mathrm{S}_{-} \mathrm{CH}_{2}-\right), 25.95(\mathrm{Ar}-$ $\left.\mathbf{C H}-\left(\mathrm{CH}_{3}\right)_{2}\right), 23.42\left(\mathrm{Ar}-\mathrm{CH}-\left(\mathrm{CH}_{3}\right)_{2}\right), 21.19\left(\mathrm{Ar}-\mathrm{CH}_{3}\right)$. 2-((4-allyl-5-(2-isopropyl-5-methylphenoxy)-4H-1,2,4triazol-3-yl)thio)-1-(4-chlorophenyl)ethanone (4i): Analytical calculated for $\mathrm{C}_{24} \mathrm{H}_{26} \mathrm{ClN}_{3} \mathrm{O}_{2} \mathrm{~S}(\%)$ : C, 63.21; H, 5.75; N, 9.21; S, 7.03; Found (\%): C, 63.40; H, 5.49; N, 9.44; S, 6.89; light yellow powder; m.p. $144-145^{\circ} \mathrm{C}$; yield $39 \%$; \%; IR(KBr) $v_{\max } \mathrm{cm}^{-1}$ : 3085 (C-H arom), 3057 (=C-H), $1678(\mathrm{C}=\mathrm{O}), 1612$ $(-\mathrm{N}=\mathrm{CH}-), 1253$ (C-O-C), $728(\mathrm{C}-\mathrm{Cl})$; MS (EI, $70 \mathrm{eV})$ : $\mathrm{m} / \mathrm{z}=456.5[\mathrm{M}+\mathrm{H}]^{+} ;{ }^{1} \mathrm{H}-\mathrm{NMR}\left(500 \mathrm{MHz}\right.$, DMSO- $d_{6}$,

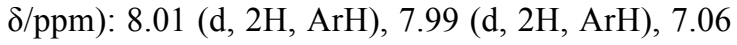
(d, 1H, ArH), 6.99 (s, 1H, ArH), 6.78 (d, 1H, ArH), $5.96\left(\mathrm{~m}, 1 \mathrm{H}, \mathrm{CH}=\mathrm{CH}_{2}\right), 5.13\left(\mathrm{~s}, 2 \mathrm{H}, \mathrm{Ar}-\mathrm{O}-\mathrm{CH}_{2}\right)$, $4.91-4.89\left(\mathrm{~d}, 1 \mathrm{H}\right.$ each, $\left.=\mathrm{CH}_{2}\right), 4.81\left(\mathrm{~d}, 2 \mathrm{H},-\mathrm{CH}_{2}-\right)$, $3.97\left(\mathrm{~s}, 2 \mathrm{H},-\mathrm{S}-\mathrm{CH}_{2}\right), 3.15$ (m, $\left.1 \mathrm{H}, \mathrm{Ar}-\mathrm{CH}-\left(\mathrm{CH}_{3}\right)_{2}\right)$, 2.31 (s, 3H, Ar- $\left.\mathrm{CH}_{3}\right), 1.10-1.09$ (d, 6H, Ar-CH$\left.\left(\mathrm{CH}_{3}\right)_{2}\right) ;{ }^{13} \mathrm{C}-\mathrm{NMR}\left(125 \mathrm{MHz}, \mathrm{DMSO}-d_{6}, \delta / \mathrm{ppm}\right)$ : $190.13(\mathrm{C}=\mathrm{O}), 154.39$ (aromatic ring $\mathrm{C}), 152.59\left(\mathrm{C}_{2^{-}}\right.$ triazole ring), $151.44\left(\mathrm{C}_{3}\right.$-triazole ring), 136.29, 133.44, 132.87 (aromatic ring $\mathrm{C}$ ), $131.30(-\mathrm{CH}=), 131.24$, $129.51,127.33,126.14,122.37$ (aromatic ring C), 118.25 $\left(=\mathrm{CH}_{2}\right), 113.41$ (aromatic ring $\left.\mathrm{C}\right), 60.43\left(-\mathrm{O}-\mathrm{CH}_{2}\right)$, $46.26\left(-\mathrm{CH}_{2}-\right), 41.17\left(-\mathrm{S}-\mathrm{CH}_{2}-\right), 25.78\left(\mathrm{Ar}-\mathrm{CH}-\left(\mathrm{CH}_{3}\right)_{2}\right)$, $23.33\left(\mathrm{Ar}-\mathrm{CH}-\left(\mathrm{CH}_{3}\right)_{2}\right), 21.29\left(\mathrm{Ar}-\mathrm{CH}_{3}\right)$.

5-(2-((4-allyl-5-(2-isopropyl-5-methylphenoxy)-4H1,2,4-triazol-3-yl)thio)acetyl)-2-hydroxybenzamide $(4 j)$ :

Analytical calculated for $\mathrm{C}_{25} \mathrm{H}_{28} \mathrm{~N}_{4} \mathrm{O}_{4} \mathrm{~S}(\%)$ : C, 62.48; H, 5.87; N, 11.66; S, 6.67; Found (\%): C, 62.71; H, $5.65 ; \mathrm{N}, 11.45$; S, 6.80; light yellow powder; m.p. $147-148^{\circ} \mathrm{C}$; yield $61 \%$; IR(KBr) $v_{\max } \mathrm{cm}^{-1}: 3350$ (N-H amide), 3098 (C-H arom), $3077(=\mathrm{C}-\mathrm{H}), 1683$ $(\mathrm{C}=\mathrm{O}$ ketone $), 1668(\mathrm{C}=\mathrm{O}$ amide $), 1612(-\mathrm{N}=\mathrm{CH}-)$, 1253 (C-O-C); MS (EI, $70 \mathrm{eV}): \mathrm{m} / \mathrm{z}=481.4[\mathrm{M}+\mathrm{H}]^{+}$; ${ }^{1} \mathrm{H}-\mathrm{NMR}\left(500 \mathrm{MHz}, \mathrm{DMSO}-d_{6}, \delta / \mathrm{ppm}\right): 12.02$ (s, $1 \mathrm{H},-\mathrm{OH}), 8.20$ (s, 2H, -CO-NH$\left.{ }_{2}\right), 8.11$ (s, 1H, ArH), 8.04 (d, 1H, ArH), 7.88 (s, 1H, ArH), 7.09 (d, 1H, ArH), 6.91 (s, 1H, ArH), 6.81 (d, 1H, ArH), 6.01 (m, $\left.1 \mathrm{H}, \mathrm{CH}=\mathrm{CH}_{2}\right), 5.23$ (s, 2H, Ar-O-CH $\left.{ }_{2}\right), 5.02$ - 4.98 $\left(\mathrm{d}, 1 \mathrm{H}\right.$ each, $\left.=\mathrm{CH}_{2}\right), 4.85\left(\mathrm{~d}, 2 \mathrm{H},-\mathrm{CH}_{2}-\right), 3.99(\mathrm{~s}, 2 \mathrm{H}$, $\left.-\mathrm{S}-\mathrm{CH}_{2}\right), 3.20\left(\mathrm{~m}, 1 \mathrm{H}, \mathrm{Ar}-\mathrm{CH}-\left(\mathrm{CH}_{3}\right)_{2}\right), 2.32$ (s, 3H, $\left.\mathrm{Ar}-\mathrm{CH}_{3}\right), 1.11-1.10\left(\mathrm{~d}, 6 \mathrm{H}, \mathrm{Ar}-\mathrm{CH}-\left(\mathrm{CH}_{3}\right)_{2}\right) ;{ }^{13} \mathrm{C}-$ NMR (125 MHz, DMSO- $\left.d_{6}, \delta / p p m\right): 190.57(\mathrm{C}=\mathrm{O})$, 167.43 (aromatic ring $\mathrm{C}$ ), $167.39(\mathrm{C}=\mathrm{O}$ amide), 154.35 (aromatic ring $\mathrm{C}), 152.69\left(\mathrm{C}_{2}\right.$-triazole ring), 151.52 $\left(\mathrm{C}_{3}\right.$-triazole ring), 136.30, 134.12, 132.91 (aromatic ring $\mathrm{C}), 131.33(-\mathrm{CH}=), 128.95,127.90,126.19,122.40$ (aromatic ring C), $118.31\left(=\mathrm{CH}_{2}\right), 117.11,115.05$, 113.38 (aromatic ring $\mathrm{C}), 60.50\left(-\mathrm{O}-\mathrm{CH}_{2}\right), 46.30$ $\left(-\mathrm{CH}_{2}-\right), 41.55\left(-\mathrm{S}-\mathrm{CH}_{2}-\right), 26.01\left(\mathrm{Ar}-\mathrm{CH}-\left(\mathrm{CH}_{3}\right)_{2}\right), 23.58$ $\left(\mathrm{Ar}-\mathrm{CH}-\left(\mathbf{C H}_{3}\right)_{2}\right), 21.36\left(\mathrm{Ar}-\mathrm{CH}_{3}\right)$.

2-((4-ethyl-5-(2-isopropyl-5-methylphenoxy)-4H-

1,2,4-triazol-3-yl)thio)acetamide (4k):

Analytical calculated for $\mathrm{C}_{17} \mathrm{H}_{24} \mathrm{~N}_{4} \mathrm{O}_{2} \mathrm{~S}(\%)$ : C, 58.59; H, 6.94; N, 16.08; S, 9.20; Found (\%): C, 58.81; H, 6.82; N, 16.24; S, 9.07; white powder; m.p. 177 $178^{\circ} \mathrm{C}$; yield $91 \%$; IR $(\mathrm{KBr}) v_{\max } \mathrm{cm}^{-1}: 3283(\mathrm{~N}-\mathrm{H}$ amide), 3050 (C-H arom), $1680(\mathrm{C}=\mathrm{O}$ amide $), 1613$ $(-\mathrm{N}=\mathrm{CH}-), 1254$ (C-O-C); MS (EI, $70 \mathrm{eV}): \mathrm{m} / \mathrm{z}=349.3$ $[\mathrm{M}+\mathrm{H}]^{+} ;{ }^{1} \mathrm{H}-\mathrm{NMR}\left(500 \mathrm{MHz}, \mathrm{DMSO}-d_{6}, \delta / \mathrm{ppm}\right)$ : $7.73\left(\mathrm{~s}, 2 \mathrm{H}, \mathrm{CO}-\mathrm{NH}_{2}\right), 7.13$ (d, 1H, ArH), 6.99 (s, 1H, ArH), 6.82 (d, 1H, ArH), 5.22 (s, 2H, Ar-O-CH CH $_{2}, 4.09$ (m, 2H, - $\mathrm{CH}_{2}$ ), 4.03 (s, 2H, $\left.-\mathrm{S}-\mathrm{CH}_{2}\right), 3.18(\mathrm{~m}, 1 \mathrm{H}$, $\left.\mathrm{Ar}-\mathrm{CH}-\left(\mathrm{CH}_{3}\right)_{2}\right), 2.31$ (s, 3H, Ar- $\left.\mathrm{CH}_{3}\right), 1.29$ (t, 3H, $\left.-\mathrm{CH}_{2}-\mathrm{CH}_{3}\right), 1.10$ - 1.09 (d, 6H, Ar-CH- $\left.\left(\mathrm{CH}_{3}\right)_{2}\right) ;{ }^{13} \mathrm{C}-$ NMR (125 MHz, DMSO- $\left.d_{6}, \delta / p p m\right): ~ 169.15(\mathrm{C}=\mathrm{O})$, 155.04 (aromatic ring $\mathrm{C}), 148.82\left(\mathrm{C}_{2}\right.$-triazole ring), 147.03 ( $\mathrm{C}_{3}$-triazole ring), 136.48, 133.90, 126.40, 122.61, 
FARMACIA, 2018, Vol. 66, 6

113.45 (aromatic ring $\mathrm{C}), 60.54\left(-\mathrm{O}-\mathrm{CH}_{2}\right), 39.17$ $\left(-\mathrm{CH}_{2}-\right), 37.21\left(-\mathrm{S}-\mathrm{CH}_{2}-\right), 26.08\left(\mathrm{Ar}-\mathrm{CH}-\left(\mathrm{CH}_{3}\right)_{2}\right), 23.31$ $\left(\mathrm{Ar}-\mathrm{CH}-\left(\mathrm{CH}_{3}\right)_{2}\right), 21.41\left(\mathrm{Ar}-\mathrm{CH}_{3}\right), 13.92\left(-\mathrm{CH}_{3}\right)$. 1-((4-ethyl-5-(2-isopropyl-5-methylphenoxy)-4H1,2,4-triazol-3-yl)thio)propan-2-one (4l):

Analytical calculated for $\mathrm{C}_{18} \mathrm{H}_{25} \mathrm{~N}_{3} \mathrm{O}_{2} \mathrm{~S}(\%)$ : C, 62.22; H, 7.25; N, 12.09; S, 9.23; Found (\%): C, 62.43; H, 7.37; N, 12.33; S, 9.11; white powder; m.p. $73-74^{\circ} \mathrm{C}$; yield $67 \%$; IR(KBr) $v_{\max } \mathrm{cm}^{-1}: 3034(\mathrm{C}-\mathrm{H}$ arom), $1710(\mathrm{C}=\mathrm{O}), 1611$ (-N=CH-), 1254 (C-O-C); MS (EI, $70 \mathrm{eV}): \mathrm{m} / \mathrm{z}=348.2[\mathrm{M}+\mathrm{H}]^{+} ;{ }^{1} \mathrm{H}-\mathrm{NMR}(500 \mathrm{MHz}$, DMSO- $\left.d_{6}, \delta / \mathrm{ppm}\right): 7.14(\mathrm{~d}, 1 \mathrm{H}, \mathrm{ArH}), 6.98(\mathrm{~s}, 1 \mathrm{H}$, ArH), 6.83 (d, 1H, ArH), 5.25 (s, 2H, Ar-O- $\mathrm{CH}_{2}$ ), 4.10 (m, 2H, - $\mathbf{C H}_{2}$ ), 4.05 (s, 2H, -S-CH $), 3.19(\mathrm{~m}, 1 \mathrm{H}$, Ar-CH- $\left.\left(\mathrm{CH}_{3}\right)_{2}\right), 2.37\left(\mathrm{~s}, 3 \mathrm{H}, \mathrm{CO}-\mathrm{CH}_{3}\right), 2.34$ (s, 3H, $\left.\mathrm{Ar}-\mathrm{CH}_{3}\right), 1.32$ (t, 3H, $\left.-\mathrm{CH}_{2}-\mathrm{CH}_{3}\right), 1.09-1.08$ (d, 6H, $\left.\mathrm{Ar}-\mathrm{CH}-\left(\mathrm{CH}_{3}\right)_{2}\right) ;{ }^{13} \mathrm{C}-\mathrm{NMR}$ (125 MHz, DMSO- $d_{6}$, $\delta / \mathrm{ppm})$ : $199.18(\mathrm{C}=\mathrm{O}), 155.11$ (aromatic ring $\mathrm{C}), 148.53$ $\left(\mathrm{C}_{2}\right.$-triazole ring), 147.99 ( $\mathrm{C}_{3}$-triazole ring), 136.51, $133.88,126.32,122.59,113.33$ (aromatic ring $\mathrm{C}$ ), $60.58\left(-\mathrm{O}-\mathrm{CH}_{2}\right), 39.21\left(-\mathrm{CH}_{2}-\right), 37.25\left(-\mathrm{S}_{-} \mathrm{CH}_{2}-\right), 26.01$ (Ar-CH- $\left.\left(\mathrm{CH}_{3}\right)_{2}\right), 23.43$ (Ar-CH- $\left.\left(\mathrm{CH}_{3}\right)_{2}\right), 21.39$ (Ar$\left.\mathrm{CH}_{3}\right), 13.88\left(-\mathrm{CH}_{3}\right)$.

2-((4-ethyl-5-(2-isopropyl-5-methylphenoxy)-4H1,2,4-triazol-3-yl)thio)-1-phenylethanone: (4m): Analytical calculated for $\mathrm{C}_{23} \mathrm{H}_{27} \mathrm{~N}_{3} \mathrm{O}_{2} \mathrm{~S}(\%)$ : C, 67.45; $\mathrm{H}, 6.65$; N, 10.26; S, 7.83; Found (\%): C, 67.76; H, $6.49 ; \mathrm{N}, 10.31 ; \mathrm{S}, 7.75$; light yellow powder; m.p. $105-106^{\circ} \mathrm{C}$; yield $40 \%$; IR(KBr) $v_{\max } \mathrm{cm}^{-1}: 3045$ (C-H arom), $1649(\mathrm{C}=\mathrm{O}), 1614(-\mathrm{N}=\mathrm{CH}-), 1238$ (C-O-C); MS (EI, $70 \mathrm{eV}): \mathrm{m} / \mathrm{z}=410.2[\mathrm{M}+\mathrm{H}]^{+} ;{ }^{1} \mathrm{H}-$ NMR (500 MHz, DMSO- $\left.d_{6}, \delta / p p m\right): 8.02(\mathrm{~d}, 2 \mathrm{H}$, ArH), 7.72 - 7.69 (t, 1H, ArH), 7.60 (d, 2H, ArH), $7.17(\mathrm{~d}, 1 \mathrm{H}, \operatorname{ArH}), 7.01(\mathrm{~s}, 1 \mathrm{H}, \operatorname{ArH}), 6.92(\mathrm{~d}, 1 \mathrm{H}$, $\mathrm{ArH}), 5.24$ (s, 2H, Ar-O-CH $\left.{ }_{2}\right), 4.12\left(\mathrm{~m}, 2 \mathrm{H},-\mathrm{CH}_{2}-\right)$, $4.08\left(\mathrm{~s}, 2 \mathrm{H},-\mathrm{S}-\mathrm{CH}_{2}\right), 3.20\left(\mathrm{~m}, 1 \mathrm{H}, \mathrm{Ar}-\mathrm{CH}-\left(\mathrm{CH}_{3}\right)_{2}\right)$, 2.36 (s, 3H, Ar- $\left.\mathrm{CH}_{3}\right), 1.35$ (t, 3H, $\left.-\mathrm{CH}_{2}-\mathrm{CH}_{3}\right), 1.09$ $1.08\left(\mathrm{~d}, 6 \mathrm{H}, \mathrm{Ar}-\mathrm{CH}-\left(\mathrm{CH}_{3}\right)_{2}\right) ;{ }^{13} \mathrm{C}-\mathrm{NMR}(125 \mathrm{MHz}$, DMSO- $\left.d_{6}, \delta / \mathrm{ppm}\right): 192.38(\mathrm{C}=\mathrm{O}), 154.93$ (aromatic ring $\mathrm{C}), 148.72\left(\mathrm{C}_{2}\right.$-triazole ring $), 148.21\left(\mathrm{C}_{3}\right.$-triazole ring), 136.52, 133.79, 130.94, 129.57, 129.14, 127.15, $126.29,122.51,113.46$ (aromatic ring C), 60.72 $\left(-\mathrm{O}-\mathrm{CH}_{2}\right), 39.16\left(-\mathrm{CH}_{2}-\right), 37.17\left(-\mathrm{S}-\mathrm{CH}_{2}-\right), 26.03$ (Ar$\left.\mathrm{CH}-\left(\mathrm{CH}_{3}\right)_{2}\right), 23.27\left(\mathrm{Ar}-\mathrm{CH}-\left(\mathrm{CH}_{3}\right)_{2}\right), 21.44\left(\mathrm{Ar}-\mathrm{CH}_{3}\right)$, $14.03\left(-\mathrm{CH}_{3}\right)$.

1-(4-chlorophenyl)-2-((4-ethyl-5-(2-isopropyl-5methylphenoxy)-4H-1,2,4-triazol-3-yl)thio)ethanone (4n):

Analytical calculated for $\mathrm{C}_{23} \mathrm{H}_{26} \mathrm{ClN}_{3} \mathrm{O}_{2} \mathrm{~S}(\%)$ : C, 62.22; H, 5.90; N, 9.46; S, 7.22; Found (\%): C, 62.46; H, 5.69 ; N, 9.28; S, 7.34; yellow powder; m.p. 142 $143^{\circ} \mathrm{C}$; yield $39 \%$; IR( $\left.\mathrm{KBr}\right) v_{\max } \mathrm{cm}^{-1}: 3055(\mathrm{C}-\mathrm{H}$ arom), 1659 (C=O), 1614 (-N=CH-), 1238 (C-O-C), 708 (C-Cl); MS (EI, $70 \mathrm{eV}): \mathrm{m} / \mathrm{z}=444.1[\mathrm{M}+\mathrm{H}]^{+}$; ${ }^{1} \mathrm{H}-\mathrm{NMR}\left(500 \mathrm{MHz}, \mathrm{DMSO}-d_{6}, \delta / \mathrm{ppm}\right): 8.10(\mathrm{~d}, 2 \mathrm{H}$, ArH), 7.63 (d, 2H, ArH), 7.16 (d, 1H, ArH), 6.98 (s, $1 \mathrm{H}, \mathrm{ArH}), 6.86(\mathrm{~d}, 1 \mathrm{H}, \mathrm{ArH}), 5.27$ (s, 2H, Ar-O-CH $)$, $4.12\left(\mathrm{~m}, 2 \mathrm{H},-\mathrm{CH}_{2^{-}}\right), 4.09$ (s, 2H, $\left.-\mathrm{S}-\mathrm{CH}_{2}\right), 3.23(\mathrm{~m}$, $\left.1 \mathrm{H}, \mathrm{Ar}-\mathrm{CH}-\left(\mathrm{CH}_{3}\right)_{2}\right), 2.39\left(\mathrm{~s}, 3 \mathrm{H}, \mathrm{Ar}-\mathrm{CH}_{3}\right), 1.38(\mathrm{t}, 3 \mathrm{H}$, $\left.-\mathrm{CH}_{2}-\mathrm{CH}_{3}\right), 1.10-1.09$ (d, 6H, Ar-CH- $\left.\left(\mathrm{CH}_{3}\right)_{2}\right) ;{ }^{13} \mathrm{C}-$ NMR (125 MHz, DMSO- $\left.d_{6}, \delta / \mathrm{ppm}\right): 192.25(\mathrm{C}=\mathrm{O})$, 154.96 (aromatic ring $\mathrm{C}), 148.91\left(\mathrm{C}_{2}\right.$-triazole ring), 147.18 ( $\mathrm{C}_{3}$-triazole ring), 136.55, 134.12, 133.91, 131.02, 129.63, 127.18, 126.35, 122.54, 113.49 (aromatic ring C), $60.69\left(-\mathrm{O}-\mathrm{CH}_{2}\right), 39.20\left(-\mathrm{CH}_{2}-\right), 37.30\left(-\mathrm{S}_{-} \mathrm{CH}_{2}-\right)$, $26.05\left(\mathrm{Ar}-\mathrm{CH}-\left(\mathrm{CH}_{3}\right)_{2}\right), 23.29\left(\mathrm{Ar}-\mathrm{CH}-\left(\mathrm{CH}_{3}\right)_{2}\right), 21.46$ $\left(\mathrm{Ar}-\mathrm{CH}_{3}\right), 14.10\left(-\mathrm{CH}_{3}\right)$.

5-(2-((4-ethyl-5-(2-isopropyl-5-methylphenoxy)-4H1,2,4-triazol-3-yl)thio)acetyl)-2-hydroxybenzamide (4o):

Analytical calculated for $\mathrm{C}_{24} \mathrm{H}_{28} \mathrm{~N}_{4} \mathrm{O}_{4} \mathrm{~S}(\%)$ : C, 61.52; H, 6.02; N, 11.96; S, 6.84; Found (\%): C, 61.75; H, 5.88; N, 12.29; S, 6.62; white powder; m.p. 133 $134^{\circ} \mathrm{C}$; yield $84 \%$; IR $(\mathrm{KBr}) v_{\max } \mathrm{cm}^{-1}: 3355(\mathrm{~N}-\mathrm{H}$ amide), 3110 (C-H arom), $1684(\mathrm{C}=\mathrm{O}$ ketone $), 1660$ ( $\mathrm{C}=\mathrm{O}$ amide), 1629 (-N=CH-), 1260 (C-O-C); MS (EI, $70 \mathrm{eV}): \mathrm{m} / \mathrm{z}=469.5[\mathrm{M}+\mathrm{H}]^{+}$; ${ }^{1} \mathrm{H}-\mathrm{NMR}(500 \mathrm{MHz}$, DMSO- $\left.d_{6}, \delta / \mathrm{ppm}\right): 12.19$ (s, $\left.1 \mathrm{H},-\mathrm{OH}\right), 8.22(\mathrm{~d}, 1 \mathrm{H}$, ArH), 8.19 (s, 2H, -CO-NH $), 8.15$ (s, 1H, ArH), 7.72 (d, 1H, ArH), 7.19 (d, 1H, ArH), 7.02 (s, 1H, ArH), 6.89 (d, 1H, ArH), 5.30 (s, 2H, Ar-O-CH $)_{2}, 4.12$ (s, 2H, $\left.-\mathrm{S}-\mathrm{CH}_{2}\right), 4.06\left(\mathrm{~m}, 2 \mathrm{H},-\mathbf{C H}_{2}-\right), 3.29$ (m, 1H, Ar-CH$\left.\left(\mathrm{CH}_{3}\right)_{2}\right), 2.38$ (s, 3H, Ar- $\left.\mathrm{CH}_{3}\right), 1.37$ (t, 3H, $\left.-\mathrm{CH}_{2}-\mathrm{CH}_{3}\right)$, 1.09 - 1.08 (d, 6H, Ar-CH- $\left.\left(\mathrm{CH}_{3}\right)_{2}\right) ;{ }^{13} \mathrm{C}-\mathrm{NMR}(125 \mathrm{MHz}$, DMSO- $\left.d_{6}, \delta / \mathrm{ppm}\right): 192.68(\mathrm{C}=\mathrm{O}), 167.73$ (aromatic ring $\mathrm{C}), 167.59(\mathrm{C}=\mathrm{O}$ amide), 154.91 (aromatic ring $\mathrm{C}), 148.56\left(\mathrm{C}_{2}\right.$-triazole ring $), 148.11\left(\mathrm{C}_{3}\right.$-triazole ring $)$, 136.59, 133.74, 132.04, 130.94, 129.57, 129.14, 128.99, $127.30,127.15,126.39,122.52,118.83,114.97,113.50$ (aromatic ring C), $60.74\left(-\mathrm{O}-\mathrm{CH}_{2}\right), 39.07\left(-\mathrm{CH}_{2}-\right)$, $37.32\left(-\mathrm{S}-\mathrm{CH}_{2}-\right), 26.10\left(\mathrm{Ar}-\mathrm{CH}-\left(\mathrm{CH}_{3}\right)_{2}\right), 23.28$ (Ar$\left.\mathrm{CH}-\left(\mathrm{CH}_{3}\right)_{2}\right), 21.50\left(\mathrm{Ar}-\mathrm{CH}_{3}\right), 14.05\left(-\mathrm{CH}_{3}\right)$.

Data obtained from the quantitative elemental analysis of the synthesized compounds was within $\pm 0.4 \%$ of the theoretical values. The structures of the intermediates $N$-substituted-acyl-thiosemicarbazides 2a-c were confirmed by their IR spectra through the presence of the absorption peaks characteristic for N-H (3316 $\left.3268 \mathrm{~cm}^{-1}\right), \mathrm{C}=\mathrm{O}\left(1681-1659 \mathrm{~cm}^{-1}\right)$ and $\mathrm{C}=\mathrm{S}(1274-$ $1266 \mathrm{~cm}^{-1}$ ) stretching vibrations and by $\mathrm{NMR}$ spectroscopy. The ${ }^{1} \mathrm{H}-\mathrm{NMR}$ spectra of compounds 2a-c displayed specific signals for $\mathrm{NH}$ protons as singlet at $7.14-7.90 \mathrm{ppm}$, and for the protons from aryl-alkyl-ether linkage as singlet at $4.98-5.23$ ppm. The ${ }^{13} \mathrm{C}$-NMR spectra of these compounds showed specific signals for the $\mathrm{C}=\mathrm{S}$ and $\mathrm{C}=\mathrm{O}$ carbons at $180.09-182.75 \mathrm{ppm}$ and $165.34-167.45 \mathrm{ppm}$, respectively. Cyclization reactions to the corresponding 3-mercapto-1,2,4-triazole compounds 3a-c were confirmed by the IR spectra through the disappearance of the absorption band characteristic for the $\mathrm{C}=\mathrm{O}$ stretching. There are two possible tautomeric isoforms corresponding for the 1,2,4-triazole-3-yl-mercapto compounds, due to the mobility of the $\mathrm{H}$ atom, which can be attached either to the nitrogen (the thione form) or to the sulphur atom (the thiol form) [24]. The 
FARMACIA, 2018, Vol. 66, 6

absence of the absorption band characteristic for the $\mathrm{N}-\mathrm{H}$ stretching vibration in the IR spectra of the 1,2,4-triazole compounds, as well as the presence of the absorption band corresponding to the $\mathrm{S}-\mathrm{H}$ stretching vibration, indicates that they exist predominantly as thiol isoform, in solid state. This fact was also confirmed by the ${ }^{1} \mathrm{H}-\mathrm{NMR}$ spectra of the above-mentioned compounds, which displayed characteristic signal for the deshielded SH proton as singlet at $13.84-14.05$ ppm, and by the ${ }^{13} \mathrm{C}-\mathrm{NMR}$ spectra, through the absence of the $\mathrm{C}=\mathrm{S}$ characteristic signal. ${ }^{1} \mathrm{H}-\mathrm{NMR}$ spectra of the target 1,2,4-triazole-3-yl-mercapto derivatives confirmed their structures, based on the disappearance of the signal characteristic for the $\mathrm{SH}$ proton and the presence of the additional signals corresponding to the $\alpha$-halocarbonyl moiety.

Molecular docking study

The target 1,2,4-triazole-3yl-mercapto derivatives and fluconazole, as control inhibitor, were docked into the catalytic site of both fungal and human lanosterol $14 \alpha$-demethylase. The predicted binding affinity for each compound, in terms of binding energy $(\Delta G)$, as well as the calculated inhibition constants $\left(\mathrm{K}_{\mathrm{i}}\right)$ for the best docking poses are presented in Table I.

Table I

Predicted binding energies $(\Delta \mathrm{G})$ and inhibition constants $\left(\mathrm{K}_{\mathrm{i}}\right)$ of the tested compounds towards the fungal and human lanosterol $14 \alpha$-demethylase

\begin{tabular}{|c|c|c|c|c|}
\hline \multirow{2}{*}{ Compound } & \multicolumn{2}{|c|}{$\Delta \mathrm{G}(\mathrm{kcal} / \mathrm{mol})$} & \multicolumn{2}{|c|}{$K_{i}(n M)$} \\
\hline & 5EQBCA & 3JUS & 5EQBCA & 3JUS \\
\hline $3 a$ & -11.05 & -9.32 & 7.95 & 147.35 \\
\hline $3 \mathbf{b}$ & -9.28 & -8.84 & 157.64 & 331.27 \\
\hline $3 c$ & -9.04 & -8.26 & 236.37 & 881.73 \\
\hline $4 a$ & -11.42 & -10.66 & 4.26 & 15.35 \\
\hline $4 b$ & -12.17 & -10.17 & 1.20 & 35.10 \\
\hline $4 c$ & -14.02 & -11.20 & 0.05 & 6.17 \\
\hline 4d & -14.20 & -11.56 & 0.04 & 3.36 \\
\hline $4 e$ & -13.58 & -12.34 & 0.11 & 0.90 \\
\hline $4 f$ & -9.90 & -9.19 & 55.36 & 183.50 \\
\hline $4 \mathrm{~g}$ & -10.39 & -9.12 & 24.21 & 206.51 \\
\hline $4 h$ & -11.36 & -10.48 & 4.71 & 20.80 \\
\hline $4 i$ & -12.66 & -10.54 & 0.52 & 18.80 \\
\hline $4 j$ & -12.00 & -10.90 & 1.60 & 10.24 \\
\hline $4 k$ & -10.22 & -9.08 & 32.26 & 220.94 \\
\hline 41 & -9.73 & -8.89 & 73.76 & 304.47 \\
\hline $4 m$ & -11.81 & -10.44 & 2.20 & 22.25 \\
\hline $4 n$ & -11.73 & -10.18 & 2.52 & 34.51 \\
\hline 40 & -12.80 & -11.85 & 0.41 & 2.06 \\
\hline fluconazole & -6.92 & -6.74 & 8463.86 & 11468.60 \\
\hline
\end{tabular}

The results of the molecular docking run demonstrated a good binding affinity towards Candida albicans lanosterol $14 \alpha$-demethylase for all the tested compounds. The synthesized 3-S-substituted-1,2,4-triazole derivatives possess binding energy values ranging from -8.13 to $-14.20 \mathrm{kcal} / \mathrm{mol}$, significantly lower than that of fluconazole $(-6.92 \mathrm{kcal} / \mathrm{mol})$. The calculated $\mathrm{K}_{\mathrm{i}}$ values showed that the tested compounds are weaker binders of the human CYP51 than of the fungal homologous, so they might be associated with a reduced mammalian toxicity.

All the synthesized compounds interact with the target enzyme through the formation of two hydrogen bonds between the phenol moiety of Tyr118 residues and the nitrogen atoms $\mathrm{N} 1$ and $\mathrm{N} 2$ of the 1,2,4-triazole heterocycle. Moreover, the ketone group of the 3mercapto-substituent forms a polar bond with the His 377-Ser378 peptide bridge. Figure 2 shows the representation of the binding patterns into the catalytic site of fungal CYP51 for the compound 4d (carbon atoms in pink), which showed the highest inhibitory activity against 5EQBCA. The non-interacting amino acid residues and tertiary structures in the foreground were removed for clarity.

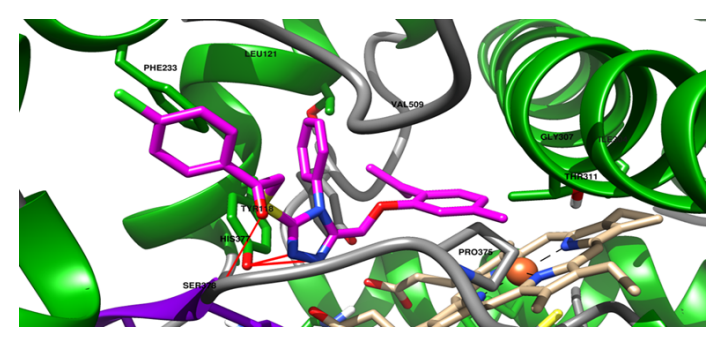

Figure 2.

The top binding conformation of compound $\mathbf{4 d}$ (carbon atoms in pink) to the active site of the fungal lanosterol $14 \alpha$-demethylase. Enzyme loops are depicted in grey and the heme carbon atoms in fawn. Protein $\alpha$ helices are depicted in green and $\beta$ sheets in purple

The docking simulation showed that all the synthesized compounds tend to interact with the amino acids from the access channel to the enzyme's catalytic site. The "T" shape of the compounds enables the insertion of the N4-substituent of the 1,2,4-triazole 
FARMACIA, 2018, Vol. 66, 6

heterocycle (phenyl, allyl or ethyl) through the amino acids from the access channel (Val509, Tyr132, Phe233). Since the accessibility to the catalytic site of CYP51 is mediated by hydrophobic interactions between the ligand and the hydrophobic amino acids residues, compounds having a large aromatic N4-substituent are better inhibitors than those having a smaller substituent. The above mentioned are supported by the smaller $K_{i}$ values of compounds N4-phenylsubstituted (4a-e), compared with those N4-allylsubstituted (4f-j) or N4-ethyl-substituted (4k-o). The orientation of the phenyl substituents (represented in Figure 3 and Figure 4 for the compound 4d) enable $\pi-\pi$ type interactions with the aromatic rings of Tyr 132 and Phe228. In addition, there might be $\pi$-cation interactions between the aromatic ring of thymol and the heme $\mathrm{Fe}^{2+}$.

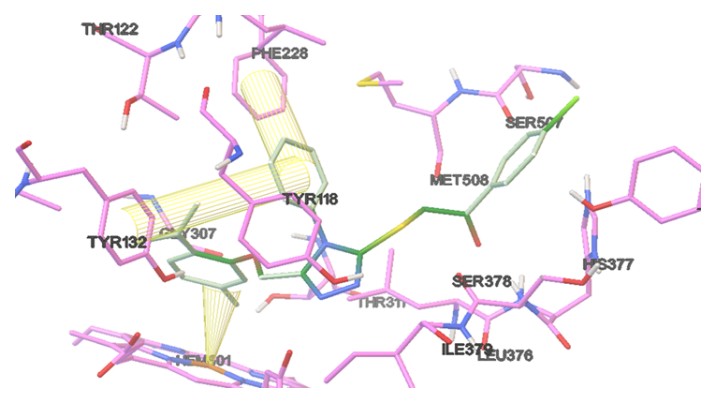

Figure 3.

Interaction of the compound $\mathbf{4 d}$ with the active site of lanosterol $14 \alpha$-demethylase; $\pi$ - $\pi$ interactions are depicted with yellow cylinders and $\pi$-cation interactions are depicted with yellow cone

The fungal CYP51 inhibition induced by the screened compounds was mediated mainly by non-covalent interactions with the amino acid residues from the access channel to the active site. As a result, mammalian toxicity raised from the cross-over inhibition of the human CYP isoforms, through the covalent-coordination of the heme $\mathrm{Fe}^{2+}$, is avoided. Based on the above observations, the synthesized compounds might be exploited for the development of novel non-competitive CYP51 inhibitors, this mechanism of action being associated with a reduction in both mammalian toxicity and fungal resistance to antifungal therapy [5].

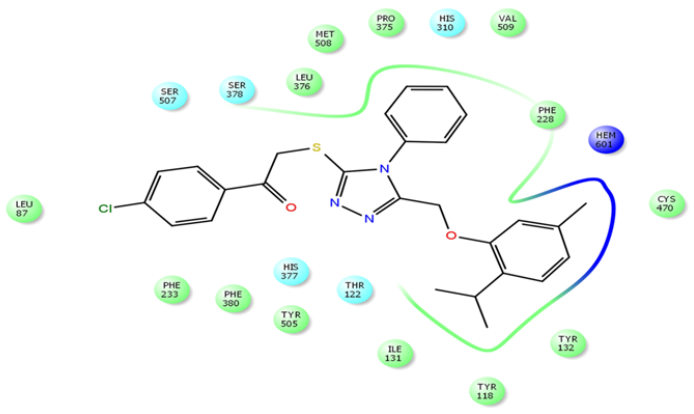

Figure 4.

A general view of the compound $\mathbf{4 d}$ inserted in the catalytic site of the fungal lanosterol $14 \alpha$-demethylase

Antifungal activity assay

The antifungal properties of the synthesized compounds were evaluated in vitro, against two Candida albicans strains (C. albicans ATCC 10231 and C. albicans ATCC 18804) and one non-albicans Candida strain (C. krusei ATCC 6258). The broth microdilution method was used for the determination of MIC and MFC values. Stock solutions $(1 \mathrm{mg} / \mathrm{mL})$ were prepared by dissolving the tested compounds and the reference antifungal, fluconazole, in sterile DMSO. The results obtained are presented in Table II.

Table II

Minimum Inhibitory Concentration $(\mu \mathrm{g} / \mathrm{mL})$ and Minimum Fungicidal Concentration $(\mu \mathrm{g} / \mathrm{mL})$ of the tested compounds

\begin{tabular}{ccccccc}
\hline \multirow{2}{*}{ Compound } & \multicolumn{2}{c}{ C. albicans (ATCC 10231) } & \multicolumn{2}{c}{ C. albicans } & (ATCC 18804) & \multicolumn{2}{c}{ C. krusei (ATCC 6258) } \\
\cline { 2 - 7 } & MIC & MFC & MIC & MFC & MIC & MFC \\
\hline $\mathbf{3 a}$ & 62.5 & 125 & 62.5 & 125 & 62.5 & 125 \\
$\mathbf{3 b}$ & $\mathbf{1 5 . 6 2}$ & $\mathbf{3 1 . 2 5}$ & $\mathbf{1 5 . 6 2}$ & $\mathbf{3 1 . 2 5}$ & 31.25 & 62.5 \\
$\mathbf{3 c}$ & 62.5 & 125 & 62.5 & 125 & 31.25 & 62.5 \\
$\mathbf{4 a}$ & 62.5 & 125 & 62.5 & 125 & 62.5 & 125 \\
$\mathbf{4 b}$ & 62.5 & 125 & 62.5 & 125 & 62.5 & 125 \\
$\mathbf{4 c}$ & 62.5 & 125 & 62.5 & 125 & 62.5 & 125 \\
$\mathbf{4 d}$ & 31.25 & 62.5 & 31.25 & 62.5 & 31.25 & 62.5 \\
$\mathbf{4 e}$ & 62.5 & 125 & 62.5 & 125 & 31.25 & 62.5 \\
$\mathbf{4 f}$ & 62.5 & 125 & 62.5 & 125 & 62.5 & 125 \\
$\mathbf{4 g}$ & 31.25 & 62.5 & 31.25 & 62.5 & 62.5 & 125 \\
$\mathbf{4 h}$ & 62.5 & 125 & 62.5 & 125 & 62.5 & 125 \\
$\mathbf{4 i}$ & 62.5 & 125 & 62.5 & 125 & 31.25 & 62.5 \\
$\mathbf{4 j}$ & 62.5 & 125 & 62.5 & 125 & 62.5 & 125 \\
$\mathbf{4 k}$ & 62.5 & 125 & 62.5 & 125 & 62.5 & 125 \\
$\mathbf{4 l}$ & 31.25 & 62.5 & 31.25 & 62.5 & 62.5 & 125 \\
$\mathbf{4 m}$ & 31.25 & 62.5 & 31.25 & 62.5 & 62.5 & 125 \\
$\mathbf{4 n}$ & 62.5 & 125 & 62.5 & 125 & 31.25 & 62.5 \\
$\mathbf{4 0}$ & 62.5 & 125 & 62.5 & 125 & 62.5 & 125 \\
fluconazole & $\mathbf{1 5 . 6 2}$ & $\mathbf{3 1 . 2 5}$ & $\mathbf{1 5 . 6 2}$ & $\mathbf{3 1 . 2 5}$ & $\mathbf{1 5 . 6 2}$ & $\mathbf{3 1 . 2 5}$ \\
\hline
\end{tabular}


MIC values registered for all the tested compounds were equal or inferior to that of the reference antifungal drug, fluconazole. The compound $\mathbf{3 b}$, having an allyl substituent attached to the N4 nitrogen of the 1,2,4-triazole nucleus, proved to be the most promising molecule, being as active as fluconazole against the Candida albicans ATCC 10231 and Candida albicans ATCC 18804 strains.

The presence of a halogenated aryl substituent attached to the 3-mercapto group proved to be favourable for the antifungal activity. The 1,2,4-triazole-3-yl-mercapto derivatives possessing a 4-Cl-phenyl moiety exhibited the stronger antifungal effect against Candida krusei ATCC 6258 strain, as compounds 4d, 4i and 4n had smaller MIC values compared with the others 1,2,4triazolyl-thioethers.

The presence of the 4-Cl-phenyl substituent also led to an improved activity against Candida albicans strains in the case of the 3-S-substituted-4-phenyl1,2,4-triazole derivatives (4a-e), compound $\mathbf{4 d}$ exhibiting the smallest MIC value in this series. Moreover, its high antifungal effect is supported by the results of the docking simulation, compound $\mathbf{4 d}$ possessing the lowest binding energy value towards the target enzyme 5EQBCA.

The substitution of the 3-mercapto group with a propan-2-one moiety seems to improve the activity of the 1,2,4-triazole-3-yl-mercapto derivatives having an N4-allyl (4f-j) or ethyl (4k-o) substituent against C. albicans strains, as shown by the MIC values of the compounds $\mathbf{4 g}$ and $\mathbf{4 l}$.

The determination of MFC confirmed the obtained MIC values. The MFC/MIC ratio values for all the tested compounds were equal to 2 , this fact suggesting a fungicidal effect [8] of the newly synthesized 1,2,4triazole derivatives.

\section{Conclusions}

A new series of 1,2,4-triazole-3-yl-mercapto derivatives were obtained in good yields, through a stepwise reaction protocol, and were investigated for their antifungal properties. The data obtained from the in silico simulation showed that the synthesized compounds are potent inhibitors of fungal lanosterol $14 \alpha$-demethylase, their affinity for the human target enzyme being significantly weaker. CYP51 inhibitory activity was not mediated by the covalent-coordination of heme $\mathrm{Fe}^{2+}$, so the synthesized compounds may have both a reduced mammalian toxicity and a lower risk that the fungal strains might develop resistance, compared to the azole class. According to the registered MIC and MFC values, the compound $\mathbf{3 b}$ proved to be the most promising molecule, being as active as the reference antifungal drug against Candida albicans strains. The target compounds (4a-o) exhibited a moderate to good activity against both Candida strains, their antifungal properties being inferior to fluconazole.
Substitution of the 3-mercapto group of the 1,2,4triazole nucleus with a 4-Cl-phenyl moiety was associated with an improved antifungal activity, as supported by the MIC values of the compounds $4 \mathbf{d}$, $\mathbf{4 i}$ and $\mathbf{4 n}$.

The results obtained encourage us to optimize further the synthesized compounds, in order to develop novel non-competitive fungal lanosterol $14 \alpha$-demethylase inhibitors.

\section{Acknowledgement}

This project was financially supported by "Iuliu Haţieganu" University of Medicine and Pharmacy Cluj-Napoca, Romania, through the PCD no. 1300/55/13.01.2017.

\section{References}

1. Ahmad A, Khan A, Akhtar F, Yousuf S, Xess I, Khan LA, Manzoor N, Fungicidal activity of thymol and carvacrol by disrupting ergosterol biosynthesis and membrane integrity against Candida. Eur J Clin Microbiol Infect Dis., 2011; 30: 41-50.

2. Ahmad A, Khan A, Manzoor N, Reversal of efflux mediated antifungal resistance underlies synergistic activity of two monoterpenes with fluconazole. Eur J Pharm Sci., 2013; 48: 80-86.

3. Arendrup MC, Patterson TF, Multidrug-resistant Candida: epidemiology, molecular mechanisms, and treatment. J Infect Dis., 2017; 216: 445-451.

4. Berman HM, Westbrook J., Feng Z., Gilliland G., Bhat T.N., Weissig H., Shindyalov I.N., Bourne P.E., The Protein Data Bank. Nucleic Acids Res., 2000; 28: 235-242.

5. Borcea AM, Marc G, Pîrnău A, Vlase L, Ionut I, Tiperciuc B, Oniga O, Synthesis and molecular docking study of some new 1,4-phenylenebisthiazoles as fungal lanosterol $14 \alpha$-demethylase inhibitors. Farmacia, 2017; 65(5): 683-689.

6. Campoy S, Adrio JL, Antifungals. Biochem Pharmacol., 2017; 133: 86-96.

7. Denning DW, Hope WW, Therapy for fungal diseases: opportunities and priorities. Trends Microbiol., 2010; 18: 195-204.

8. Hafidh RR, Abdulamir AS, Vern LS, Abu Bakar F, Abas F, Jahanshiri F, Sekawi Z, Inhibition of growth of highly resistant bacterial and fungal pathogens by a natural product. Open Microbiol J., 2011; 5: 96-106.

9. Kharb R, Shahar Yar M, Sharma PC, New insights into chemistry and anti-infective potential of triazole scaffold. Curr Med Chem., 2011; 18: 3265-3297.

10. Küçükgüzel $G$, Çikla-Süzgün $P$, Recent advances bioactive 1,2,4-triazole-3-thiones. Eur J Med Chem., 2015; 97: 830-870.

11. Kulabaş N, Tatar E, Bingöl Özakpınar Ö, Özsavci D, Pannecouque C, De Clercq E, Küçükgüzel I, Synthesis and antiproliferative evaluation of novel 2-(4H-1,2,4-triazole-3-ylthio)acetamide derivatives as inducers of apoptosis in cancer cells. Eur J Med Chem., 2016; 121: 58-70.

12. Lin GS, Duan WG, Yang LX, Huang M, Lei FH, Synthesis and antifungal activity of novel myrtenal- 
based 4-methyl-1,2,4-triazole-thioethers. Molecules, 2017; 22: 1-10.

13. Li YH, Zhang B, Yang HK, Li Q, Diao PC, You WW, Zhao PL, Design, synthesis, and biological evaluation of novel alkylsulfanyl-1,2,4-triazoles as cis-restricted combretastatin A-4 analogues. Eur J Med Chem., 2017; 125: 1098-1106.

14. Marc G, Ionuț I, Pîrnău A, Vlase L, Vodnar DC, Duma M, Tiperciuc B, Oniga O, Microwave assisted synthesis of 3,5-disubstituted tiazolidine-2,4-diones with antifungal activity. Design, synthesis, virtual and in vitro antifungal screening. Farmacia, 2017; 65(3): 414-422.

15. Morris G, Huey R, AutoDock4 and AutoDockTools4: automated docking with selective receptor flexibility. J Comput Chem., 2009; 30: 2785-2791.

16. Pettersen EF, Goddard TD, Huang CC, Couch GS, Greenblatt DM, Meng EC, Ferrin TE, UCSF Chimera A visualization system for exploratory research and analysis. J Comput Chem., 2004; 25: 1605-1612.

17. Rao A, Zhang Y, Muend S, Rao R, Mechanism of antifungal activity of terpenoid phenols resembles calcium stress and inhibition of the TOR pathway. Antimicrob Agents Chemother., 2010; 54: 5062-5069.

18. Rex JH, Alexander BD, Andes D, Arthington-Skaggs B, Brown SD, Chaturvedi V, Ghannoum MA, EspinelIngroff A, Knapp CC, Ostrosky-Ziechner L, Pfaller MA, Sheehan DJ, Walsh TJ, Reference method for broth dilution antifungal susceptibility testing of yeasts. Third Edition. Clin Lab Stand Inst., 2008; 1-25.

19. Said SA, Synthesis of some new 2-(2-isopropyl-5methylphenoxymethyl)-5-aryl-1,3,4-oxadiazoles. Rafidain J Sci., 2006; 17: 52-58.

20. Salci TP, Negri M, Abadio AKR, Svidzinski TIE., Kioshima ES, Targeting Candida spp. to develop antifungal agents. Drug Discov Today, 2018; 0: 1-13.
21. Stana A, Enache A, Vodnar DC, Nastasă C, Benedec D, Ionuț I, Login C, Marc G, Oniga O, Tiperciuc B, New thiazolyl-triazole Schiff bases: synthesis and evaluation of the anti-Candida potential. Molecules, 2016; 21: 1-19.

22. Strushkevich N, Usanov SA, Park HW, Structural basis of human CYP51 inhibition by antifungal azoles. J Mol Biol., 2010; 397: 1067-1078.

23. Sun B, Huang W, Liu M, Evaluation of the combination mode of azoles antifungal inhibitors with CACYP51 and the influence of site-directed mutation. $J \mathrm{Mol}$ Graph Model, 2017; 73: 157-165.

24. Tiperciuc B, Zaharia V, Colosi I, Moldovan C, Crișan O, Pîrnău A, Vlase L, Duma M, Oniga O, Synthesis and evaluation of antimicrobial activity of some new hetaryl-azoles derivatives obtained from 2-aryl-4methylthiazol-5-carbohydrazides and isonicotinic acid hydrazide. J Heterocycl Chem., 2012; 49: 14071414.

25. Zhou $\mathrm{CH}$, Wang $\mathrm{Y}$, Recent researches in triazole compounds as medicinal drugs. Curr Med Chem., 2012; 19: 239-280.

26. Yates CM, Garvey EP, Shaver SR, Schotzinger RJ, Hoekstra WJ, Design and optimization of highlyselective, broad spectrum fungal CYP51 inhibitors. Bioorganic Med Chem Lett, 2017; 27: 3243-3248.

27. Wiederhold N, Antifungal resistance: current trends and future strategies to combat. Infect Drug Resist., 2017; 10: 249-259.

28. Wiederhold NP, Patterso TF, What's new in antifungals: an update on the in-vitro activity and invivo efficacy of new and investigational antifungal agents. Curr Opin Infect Dis., 2015; 28: 539-546. 